\title{
SCATTERING THEORY FOR AUTOMORPHIC FUNCTIONS
}

\author{
BY PETER D. LAX AND RALPH S. PHILLIPS ${ }^{1}$
}

\begin{abstract}
This paper is an expository account of our 1976 monograph [6] on Scattering theory for automorphic functions. Several improvements have been incorporated: a more direct proof of the meromorphic character of the Eisenstein series, an explicit formula for the translation representations and a simpler derivation of the spectral representations. Our hyperbolic approach to the Selberg trace formula is also included.
\end{abstract}

1. Introduction. In 1972 Faddeev and Pavlov [2] discovered a revealing connection between the harmonic analysis of functions automorphic with respect to a discrete subgroup of $\operatorname{SL}(2, R)$ and the Lax-Phillips scattering theory as applied to the non-Euclidean wave equation. Their work is based on the spectral theory for the Laplace-Beltrami operator previously developed by Faddeev [1] using elliptic arguments. In our 1976 monograph [6] we redid the Faddeev-Pavlov paper entirely within the framework of our theory, basing our development on the non-Euclidean wave equation. We obtained new treatments for (i) the spectral theory of the Laplace-Beltrami operator over noncompact domains of finite area; (ii) the meromorphic character of the Eisenstein series over the whole complex plane; and (iii) a new form of the Selberg trace formula.

In this paper we sketch a revised version of our monograph including a more direct proof of the meromorphic character of the Eisenstein series, an explicit formula for the translation representations and a simpler derivation of the spectral representations.

The harmonic analysis of automorphic functions has been extensively studied; references to the pertinent parts of this theory are contained in our monograph. We recall that the Poincare plane $\Pi$, that is the upper half plane

$$
w=x+i y, \quad y>0,
$$

serves as a model for a non-Euclidean geometry in which the motions are given by the group $G$ of fractional linear transformations:

$$
w \rightarrow \frac{a w+b}{c w+d}
$$

where $a, b, c, d$ are real and

$$
a d-b c=1
$$

Received by the editors August 1, 1979.

AMS (MOS) subject classifications (1970). Primary

${ }^{1}$ The work of both authors was supported in part by the National Science Foundation, the first author under Grant No. MCS-76-07039 and the second under Grant No. MCS-77-04908 A 01. (C) 1980 American Mathematical Society 0002-9904/80/0000-0100/\$09.75 
$G$ is isomorphic with $\operatorname{SL}(2, R) / \pm I$. The Riemannian metric

$$
\frac{d x^{2}+d y^{2}}{y^{2}}
$$

is invariant under this group of motions. The invariant $L_{2}$ form is

$$
\iint u^{2} \frac{d x d y}{y^{2}}
$$

The invariant Dirichlet form is

$$
\iint\left(u_{x}^{2}+u_{y}^{2}\right) d x d y .
$$

The corresponding Laplace-Beltrami operator

$$
L_{0}=y^{2} \Delta=y^{2}\left(\partial_{x}^{2}+\partial_{y}^{2}\right)
$$

is then clearly invariant. It turns out that the operator $L$ defined as

$$
L=L_{0}+1 / 4 \text {, }
$$

also invariant, has more useful analytic properties, as will be seen in what follows.

A subgroup $\Gamma$ of $G$ is called discrete if the identity is not a limit point of $\Gamma$. A fundamental domain $F$ for a discrete subgroup $\Gamma$ is a suludomain of $\Pi$ such that every point of $\Pi$ can be carried into a point of $\bar{F}$ by a transformation in $\Gamma$ and no point of $F$ is carried into another point of $F$ by such a transformation. A function $f$ defined on $\Pi$ is called automorphic with respect to $\Gamma$ if

$$
f(\gamma w)=f(w)
$$

for all $\gamma$ in $\Gamma$. Because of (1.8) an automorphic function is completely determined by its values on $\bar{F}$. If $f$ is continuous then (1.8) imposes a relation between values of $f$ at those pairs of boundary points of $F$ which can be mapped into each other by some $\gamma$ in $\Gamma$. If $f$ is $C^{1}$, then (1.8) imposes a similar relation on the first derivatives of $f$ at such pairs of boundary points.

The Laplace-Beltrami operator, being invariant, maps automorphic functions into automorphic functions. Alternatively we can consider automorphic functions as being defined on $F$ : In this case we introduce the space $L_{2}(F)$ of functions on $F$ square integrable with respect to the invariant measure and define $L$ as the selfadjoint extension of the differential operator defined by $(1.7)^{\prime}$, subject to the above mentioned boundary conditions.

We shall study the spectral properties of $L$ by means of the non-Euclidean wave equation

$$
u_{t t}=y^{2} \Delta u+u / 4=L u .
$$

This turns out to be a convenient analytic tool. As Semenov-Tian-Shansky [11] has recently shown, this equation also has an intrinsic meaning in the Lie algebra framework for problems of this kind. Indeed many of the classical concepts previously introduced in the study of these problems (see Kubota [4]) appear in a natural way in the scattering theory setting for the nonEuclidean wave equation. In particular the translation representations for the wave equation are closely related to the Radon transform associated with 
symmetric spaces as well as the theta series employed by Kubota in the study of Eisenstein series.

Perhaps the simplest discrete subgroup of $G$ is the modular group, consisting of the fractional linear transformations with $a, b, c, d$ integers satisfying (1.3). A convenient fundamental domain for this subgroup is the geodesic triangle

$$
F:-1 / 2<x<1 / 2, \quad y>\sqrt{1-x^{2}} .
$$

For the sake of simplicity, most of the detailed discussion in this paper will be carried out for this spectral subgroup.

We conclude this introduction with a brief description of our abstract scattering theory and its application to the classical wave equation in Euclidean space. For details we refer to our book [5]. The theory concerns a one-parameter group of unitary operators $U(t)$ acting on a Hilbert space $\mathcal{H C}$ for which there exist certain subspaces $\mathscr{D}_{-}$and $\mathscr{D}_{+}$, called incoming and outgoing with the following properties:

(i) $U(t) \mathscr{D}_{-} \subset \mathscr{D}_{-}$for $t \leqslant 0$ and $U(t) \mathscr{D}_{+} \subset \mathscr{D}_{+}$for $t \geqslant 0$,

(ii) $\cap U(t) \mathscr{Q}_{-}=\{0\}=\bigcap U(t) \mathscr{D}_{+}$,

$$
\overline{U U(t) \mathscr{Q}_{-}}=\mathscr{H}=\overline{U U(t)^{\mathscr{Q}}+} \text {. }
$$

An example of an outgoing subspace is given by the following: $\mathcal{H C}=$ $L_{2}(\mathbf{R}, \Re), \Re$ some auxiliary Hilbert space, $U(t)$ translation to the right by an amount $t$, and $\mathscr{D}_{+}=L_{2}\left(\mathbf{R}_{+}, \mathscr{T}\right)$. It turns out that this class of examples exhausts all possibilities since every Hilbert space $\mathcal{H}$ with a unitary group for which there is an outgoing subspace can be represented as above, essentially uniquely; this is called the outgoing translation representation of $\mathcal{H}$. Corresponding to an incoming subspace there is an incoming translation representation in which $\mathscr{D}_{-}$maps onto $L_{2}\left(\mathbf{R}_{-}, \mathscr{T}\right)$.

In the applications of the theory we are about to describe, $t$ represents time and the group $U(t)$ describes the propagation of waves. The significance of incoming and outgoing subspaces for wave propagation is that they furnish an asymptotic description of signals in the remote past and distant future respectively. The scattering operator $S$ relates the incoming translation representer of a given data to its outgoing representer. Thus $S$ gives a description of the scattering process, i.e. it relates directly the asymptotic behavior of waves for large negative and positive times.

$S$ maps $L_{2}(\mathbf{R}, \mathscr{N})$ onto itself; since it relates two translation representations it is unitary and commutes with translation. Therefore $S$ is convolution with an operator valued distribution $(\Re \rightarrow \Re)$ called the scattering function and denoted as $S(t)$ (cf. [7]).

This approach is particularly fruitful when $\mathscr{D}_{-}$and $\mathscr{D}_{+}$are orthogonal to each other; in this case $S$ is a so-called causal operator, in the sense that the scattering function $S(t)=0$ for $t>0$. In this case the Fourier transform of $S(t)$, called the scattering matrix and denoted as $\delta(z)$, is analytic in the lower half plane. It turns out that many important properties of the scattering 
process can be related to the behavior of the meromorphic continuation of $\delta(z)$ into the upper half plane.

This connection can be studied most directly through the one-parameter semigroup of operators $Z(t)$, carved out of the group $U(t)$ as follows:

$$
Z(t)=P_{+} U(t) P_{-}, \quad t \geqslant 0,
$$

where $P_{+}$and $P_{-}$are the orthogonal projections that remove the components of data that belong to $\mathscr{D}_{+}$and $\mathscr{D}_{-}$, respectively. It is easy to show that $Z(t)$, $t \geqslant 0$, annihilates $\mathscr{Q}_{+}$and $\mathscr{Q}_{-}$and acts as a semigroup of operators on $\mathscr{K}=\mathcal{H} \Theta\left(\mathscr{D}_{-} \oplus \mathscr{D}_{+}\right)$. The resolvent of the infinitesimal generator of $Z$ turns out to be intimately related to the scattering matrix.

Before returning to the automorphic case we would like to indicate how these ideas are used to study wave propagation in Euclidean space in the presence of an obstacle $\theta$. In the exterior $\mathcal{E}$ of the obstacle the signal satisfies the Euclidean wave equation:

$$
u_{t t}=\Delta u \text {. }
$$

The interaction of the wave with the obstacle is described as a boundary condition on $u$; if the interaction conserves the energy contained in the wave, the boundary condition makes $\Delta$ a selfadjoint operator on $L_{2}(\mathcal{E})$. Typical of such boundary conditions are $u=0$ (or $\partial_{n} u=0$ ) on $\partial \theta$.

The underlying Hilbert space is the space of initial data $\left\{u, u_{t}\right\}$ defined on $\mathcal{E}$ and normed by the energy norm:

$$
E_{\mathscr{E}}(u)=-(u, \Delta u)_{\mathscr{E}}+\left(u_{t}, u_{t}\right)_{\mathscr{\xi}},
$$

where $(\text {, })_{\mathscr{E}}$ denotes the $L_{2}$ inner product over $\mathscr{E}$. Since $\Delta$ is selfadjoint, $E_{\mathscr{E}}(u)$ is independent of $t$ for solutions $u$ of (1.14) satisfying the boundary conditions. This shows that the operator $U(t)$ relating initial data at time 0 to data at time $t$ is an isometry; since the initial-boundary value problem is reversible in time and can be solved for a dense set of data, the operators $U(t)$ are actually unitary and form a group.

We call a solution $u$ outgoing if $u(x, t)=0$ at all points $x$ whose distance from the boundary is less than $t$; an incoming solution is described similarly with $t$ replaced by $-t . \mathscr{D}_{-}$and $\mathscr{D}_{+}$are defined as the initial data of incoming and outgoing solutions, respectively. Properties (i) and (ii) can be immediately verified; property (iii) lies considerably deeper and says essentially that all incoming signals eventually become outgoing signals after a complicated process of reflection from the boundary.

Roughly speaking, the same picture can be painted of automorphic waves over noncompact fundamental domains. Signals coming in from or going out to infinity can be defined analogously. Again one of the main results to be proved is that all incoming signals are eventually turned into outgoing signals. The analysis is complicated by the possible presence of standing waves due to a rather rich point spectrum, and of exponentially increasing solutions due to the indefiniteness of the energy form. A treatment of the corresponding free space problem can be found in [8].

2. The non-Euclidean wave equation. The first step in our analysis consists in finding a suitable Hilbert space setting for the initial value problem 
associated with the non-Euclidean wave equation:

$$
\begin{gathered}
u_{t t}=L u, \\
u(x, 0)=f_{1}(x), \quad u_{t}(x, 0)=f_{2}(x) .
\end{gathered}
$$

For a more complete treatment we refer the reader to $[6, \S 5]$.

In terms of the $L_{2}(F)$ inner product $(,)_{F}$, the energy form for the wave equation is

$$
E_{F}(u)=-(u, L u)_{F}+\left(u_{t}, u_{t}\right)_{F} \text {. }
$$

Since $L$ is selfadjoint in $L_{2}(F), E_{F}(u)$ is independent of $t$ for automorphic solutions of (2.1). In general $E_{F}$ is indefinite for automorphic data; this can be seen if we bring (2.2) into a more symmetric form by an integration by parts:

$$
E_{F}(u)=\iint_{F}\left\{\left|u_{x}\right|^{2}+\left|u_{y}\right|^{2}-\frac{|u|^{2}}{4 y^{2}}+\frac{\left|u_{t}\right|^{2}}{y^{2}}\right\} d x d y .
$$

The indefiniteness introduces complications analogous to those produced by bound states in the theory of scattering for quantum mechanics (see [5, Chapter 6]).

It is useful at this point to write the energy form in a way which depends on the shape of the fundamental domain $F$. In the case of the modular group with fundamental domain described in (1.10) we split $F$ up into two parts:

$$
F_{0}=F \cap\{y \leqslant a\},
$$

which is compact, and a neighborhood of infinity

$$
F_{1}=F \cap\{Y>a\}
$$

here $a$ is restricted to $b e>1$. Writing (2.2) as the sum of integrals over $F_{0}$ and $F_{1}$ an integrating by parts over $F_{1}$, the energy form becomes

$$
\begin{aligned}
E_{F}(u)= & \iint_{F_{0}}\left\{\left|u_{x}\right|^{2}+\left|u_{y}\right|^{2}-\frac{|u|^{2}}{4 y^{2}}+\frac{\left|u_{t}\right|^{2}}{y^{2}}\right\} d x d y \\
& +\iint_{F_{1}}\left\{\left|u_{x}\right|^{2}+y\left|\partial_{y} \frac{u}{\sqrt{y}}\right|^{2}+\frac{\left|u_{t}\right|^{2}}{y^{2}}\right\} d x d y \\
& -\frac{1}{2 a} \int_{-1 / 2}^{1 / 2}|u(x, a)|^{2} d x .
\end{aligned}
$$

From now on we omit the subscript $F$ except where it is needed for clarity.

Next we introduce a new form which is very close to (2.2)" but which has the advantage of being positive definite:

$$
G(u)=E(u)+2 K(u),
$$

where

$$
K(u)=\iint_{F_{0}} \frac{|u|^{2}}{y^{2}} d x d y
$$


Notice that for data with support in $F_{1}$ the $E$ and $G$ forms are the same. It can be shown by simple estimates that $G$ is positive definite and equivalent with

$$
\begin{aligned}
G^{\prime}(u)= & \iint_{F_{0}}\left\{\left|u_{x}\right|^{2}+\left|u_{y}\right|^{2}+\frac{|u|^{2}}{y^{2}}\right\} d x d y \\
& +\iint_{F_{1}}\left\{\left|u_{x}\right|^{2}+y\left|\partial_{y} \frac{u}{\sqrt{y}}\right|^{2}\right\} d x d y+\iint_{F} \frac{\left|u_{t}\right|^{2}}{y^{2}} d x d y .
\end{aligned}
$$

By Rellich's theorem, $K$ is compact with respect to $G^{\prime}$.

We denote by $\mathcal{H}_{G}$ the completion in the $G$-norm of the space of $C^{\infty}$ automorphic data with compact support in $F$. Since the $K$-form is compact with respect to the $G$-form it follows that the $E$ and $G$-forms are equivalent on any closed subspace of $\mathcal{H}_{G}$ on which $E$ is positive.

In order to treat $u$ and $u_{t}$ on an equal footing we rewrite the wave equation in component form as

$$
u_{t}=v, \quad v_{t}=L u
$$

or in matrix notation as

$$
V_{t}=A V
$$

where

$$
V=\left(\begin{array}{l}
u \\
v
\end{array}\right) \text { and } A=\left(\begin{array}{ll}
0 & I \\
L & 0
\end{array}\right)
$$

We take as $D(A)$, the domain of $A$, the set of data $(u, v)$ for which both $(u, v)$ and $(v, L u)$ belongs to $\mathcal{H}_{G}, L u$ being defined in the weak sense. It can be shown that $A$ generates on $\mathcal{H}_{G}$ a group of bounded operators $U(t)$ which grow exponentially in the $G$-norm but are unitary with respect to the indefinite energy form $E$. Since (2.1) is hyperbolic, signals carried by solutions of (2.1) propagate with non-Euclidean speed $\leqslant 1$.

Of particular interest to us are certain subspaces of $\mathcal{H}_{G}$ which we call incoming and outgoing spaces and denote by $\mathscr{D}_{-}$and $\mathscr{D}_{+}$, respectively. For the modular group and $F$ of the form (1.10) these subspaces are the initial data for solutions of (2.1) with support in $F_{1}$ and which are independent of $x$ for $t<0(t>0)$ in the case of $\mathscr{D}_{-}\left(\mathscr{D}_{+}\right)$; see $\$ 6$ of [6]. A solution of (2.1) which is independent of $x$ satisfies the differential equation

$$
u_{t t}=y^{2} \Delta u+u / 4 \text {. }
$$

The change of variables

$$
s=\log y, \quad v=u / \sqrt{y},
$$

transforms (2.8) into the classsical wave equation:

$$
v_{t t}=v_{s s}
$$

whose general solution is

$$
v=l(s+t)+r(s-t)
$$

The first term on the right corresponds to a wave traveling to the left, the 
second term to a wave traveling to the right. The corresponding incoming and outgoing solutions of (2.1) are of the form

$$
\begin{gathered}
u(w, t)=y^{1 / 2} \varphi\left(y e^{t}\right), \\
u(w, t)=y^{1 / 2} \varphi\left(y e^{-t}\right) ;
\end{gathered}
$$

here $\varphi$ is chosen to be $C^{\infty}$ and vanishing for $y \leqslant a$.

We define the incoming and outgoing subspaces $\mathscr{D}_{ \pm}$as the closure in $\mathcal{H}_{G}$ of the initial data of the above respective incoming and outgoing solutions:

$$
\begin{aligned}
& \mathscr{D}_{-}=\operatorname{closure}\left\{y^{1 / 2} \varphi(y), y^{3 / 2} \varphi^{\prime}(y)\right\}, \\
& \mathscr{D}_{+}=\operatorname{closure}\left\{y^{1 / 2} \varphi(y),-y^{3 / 2} \varphi^{\prime}(y)\right\},
\end{aligned}
$$

$\varphi$ in $C^{\infty}$, zero for $y \leqslant a, \varphi^{\prime}=\partial_{y} \varphi$.

It is clear from this that $\mathscr{D}_{ \pm}$have the properties, see (1.11):

$$
\begin{aligned}
& \text { (i) } U(t) \mathscr{D}_{ \pm} \subset \mathscr{D}_{ \pm} \text {for } t \gtrless 0, \\
& \text { (ii) } \cap U(t) \mathscr{D}_{-}=\{0\}=\bigcap U(t)^{\mathscr{D}_{+}} .
\end{aligned}
$$

The change of variables (2.9) maps the energy form $E$ defined by (2.2) into the classical energy form $\int\left\{\left|v_{s}\right|^{2}+\left|v_{t}\right|^{2}\right\} d s$ for (2.10). In these variables a trivial calculation shows that

$$
\mathscr{D}_{-} \text {and } \mathscr{D}_{+} \text {are } E \text {-orthogonal. }
$$

Since data in $\mathscr{D}_{-}$and $\mathscr{D}_{+}$are zero in $F_{0}$, it follows that $\mathscr{D}_{-}$and $\mathscr{D}_{+}$are also $\boldsymbol{G}$-orthogonal.

We denote the zero Fourier coefficient of $f$ with respect to $x$ by $f^{(0)}$ :

$$
f^{(0)}(y)=\int_{-1 / 2}^{1 / 2} f(x, y) d x, \quad y>a .
$$

Suppose the data $f=\left(f_{1}, f_{2}\right)$ in $\mathcal{H}_{G}$ is $E$-orthogonal to $\mathscr{D}_{-}$; it is easy to show by using the definition (2.13) (2.2)" that the components of $f^{(0)}$ satisfy

$$
f_{2}^{(0)}=-y^{3 / 2} \partial_{y}\left(\frac{f_{1}^{(0)}}{\sqrt{y}}\right) \text { for } y>a .
$$

Similarly if it is $E$-orthogonal to $\mathscr{D}_{+}$then

$$
f_{2}^{(0)}=y^{3 / 2} \partial_{y}\left(\frac{f_{1}^{(0)}}{\sqrt{y}}\right) \text { for } y>a .
$$

If $f$ in $\mathcal{H}_{G}$ is orthogonal to both $\mathscr{D}_{-}$and $\mathscr{D}_{+}$then (2.16) implies that the zero Fourier coefficient of $f$ is of the form

$$
f^{(0)}=(c \sqrt{y}, 0) \text { for } y \geqslant a .
$$

We denote the set of all such data by $\mathcal{K}$ :

$$
\mathscr{K}=\mathcal{H}_{G} \ominus\left(\mathscr{Q}_{-} \oplus \mathscr{D}_{+}\right) \text {. }
$$

The next theorem is the basic technical tool in this application of our scattering theory. 
THEOREM 2.1. For every $\lambda$ in the resolvent set of $A$, the resolvent operator $(\lambda I-A)^{-1}$ maps the unit ball in $\mathcal{K}$ into a compact subset of $\mathcal{H}_{G}$.

We give a new proof of this theorem in the appendix.

Corollary 2.2. A has at most a denumerable set of eigenfunctions in $\mathcal{K}$ and the corresponding eigenvalues are discrete.

3. The associated semigroup of operators. In this section the group of operators $U$ and the incoming and outgoing subspaces are combined to form a semigroup of operators which plays an important role in our theory.

To this end we introduce the orthogonal projections $\boldsymbol{P}_{-}$and $\boldsymbol{P}_{+}$which remove the $\mathscr{D}_{-}$and $\mathscr{D}_{+}$components, respectively. Again since the $G$ and $E$-forms are the same for $y>a, P_{ \pm}$is orthogonal with respect to both forms. Notice also that since $\mathscr{Q}_{-}$and $\mathscr{Q}_{+}$are orthogonal,

$$
P=P_{-}+P_{+}
$$

is the orthogonal projection on $\mathscr{K}$. Using the relations (2.16) and (2.17) it is easy to verify for any $f$ in $\mathcal{H}_{G}$ that $g=P f$ can be decribed as follows:

$$
\left.\begin{array}{l}
g=f \quad \text { for } y<a, \\
g_{1}(w)=f_{1}(w)-f_{1}^{(0)}(y)+\left(\frac{y}{a}\right)^{1 / 2} f_{1}^{(0)}(a) \\
g_{2}(w)=f_{2}(w)-f_{2}^{(0)}(y)
\end{array}\right\} \text { for } y \geqslant a .
$$

We now set

$$
Z(t)=P_{+} U(t) P_{-} \quad \text { for } t \geqslant 0 \text {. }
$$

THEOREM 3.1. The operators $Z(t), t \geqslant 0$, form a strongly continuous semigroup of operators on $\mathscr{K} . Z(t)$ annihilates both $\mathscr{D}_{-}$and $\mathscr{D}_{+}$.

A proof of this theorem can be based entirely on the properties $(2.14)_{i}$ and (2.14)' (see Theorem 2.7 of [6]).

We denote the infinitesimal generator of $Z(t)$ by $B$. We have noted earlier that $U$ grows at most exponentially:

$$
\|U(t)\| \leqslant C e^{\omega|t|}
$$

Obviously $Z$ satisfies an analogous inequality:

$$
\|Z(t)\| \leqslant C e^{\omega t} \text {. }
$$

Consequently for $f$ in $\mathscr{K}$ and $\operatorname{Re} \lambda>\omega$ we can write

$$
\begin{aligned}
(\lambda I-B)^{-1} f & =\int_{0}^{\infty} e^{-\lambda t} Z(t) f d t \\
& =P_{+} \int_{0}^{\infty} e^{-\lambda t} U(t) f d t=P_{+}(\lambda I-A)^{-1} f
\end{aligned}
$$

Combining this with Theorem 2.1 we can now state

THEOREM 3.2 The resolvent of $B$ is compact on $\mathscr{K}$.

COROLlary 3.3. The resolvent of $B$ is meromorphic in the entire complex plane and $B$ has a pure point spectrum. 
Since by (2.14) $U(t) \mathscr{D}_{+} \subset \mathscr{Q}_{+}$for $t \geqslant 0$, we see that

$$
P_{+} U(t)\left(I-P_{+}\right)=0 \text {. }
$$

Hence for $f$ orthogonal to $\mathscr{D}_{-}$we have

$$
Z(t) P_{+} f=P_{+} U(t) P_{+} f=P_{+} U(t) f .
$$

If in addition $f$ belongs to the domain of $A$, then we can differentiate the above relation at $t=0$ and obtain

$$
B P_{+} f=P_{+} A f
$$

a relation we shall use in $\S 4$.

4. The Eisenstein series and its analytic continuation. We are now ready to construct the generalized eigenfunctions of $\boldsymbol{A}$ associated with the continuous part of its spectrum. It is readily verified that for any complex $z$ the data

$$
h(w)=\left\{y^{1 / 2+i z}, i z y^{1 / 2+i z}\right\}
$$

locally satisfies

$$
A h=i z h .
$$

Since $A$ is invariant, the function $h^{\gamma}(w)=h(\gamma w)$ satisfies

$$
A h^{\gamma}=i z h^{\gamma}
$$

for every $\gamma$ in $G$. Since $h$ is by definition independent of $x$, it is automorphic with respect to the subgroup $\Gamma_{\infty}$ consisting of $\gamma: w \rightarrow w+n, n$ integer; that is

$$
h(\gamma w)=h(w), \quad \gamma \in \Gamma_{\infty} .
$$

In order to construct an automorphic function out of $h$ for the entire modular group $\Gamma$ we have to sum $h(\gamma w)$ over all right cosets of $\Gamma$ modulo $\Gamma_{\infty}$. This sum is the Eisenstein series:

$$
e(w, z)=\sum_{\gamma \in \Gamma_{\infty} \backslash \Gamma} h(\gamma w) .
$$

It is well known that this series converges for $\operatorname{Im} z<-1 / 2$; except for the first term $h(w)$, the series converges in the $G$-norm for such $z$. Since $(A-i z)$ annihilates each term we conclude that

$$
(A-i z) e=0
$$

in the sense of distributions for $\operatorname{Im} z<-1 / 2$. It follows from elliptic regularity theory that $e$ is $C^{\infty}$ and satisfies (4.5) pointwise.

Recall the definition (2.7)" of $A$ as $\left(\begin{array}{ll}0 & I \\ L\end{array}\right)$; it follows from this and (4.5) that the first component $e_{1}$ of $e$ satisfies the equation

$$
L e_{1}=-z^{2} e_{1} \text {. }
$$

Integrating this with respect to $x$ gives the following ordinary differential equation for the zero Fourier coefficient of $e_{1}$ :

$$
\left(y^{2} \partial_{y}^{2}+1 / 4\right) e_{1}^{(0)}=-z^{2} e_{1}^{(0)} .
$$

The solutions to this equation are superpositions of $y^{\kappa}$, where $\kappa$ satisfies

$$
\kappa(\kappa-1)+1 / 4=-z^{2},
$$


that is $\kappa=1 / 2 \pm i z$. Since by (4.5), $e_{2}=i z e_{1}$ we deduce for $\operatorname{Im} z<-1 / 2$ that the zero Fourier coefficient of $e$ defined by (4.4) is of the form

$$
e^{(0)}(y, z)=\left\{y^{1 / 2+i z}, i z y^{1 / 2+i z}\right\}+s(z)\left\{y^{1 / 2-i z}, i z y^{1 / 2-i z}\right\},
$$

where $s(z)$ is some function of $z$. A straightforward calculation, using (4.4), shows that

$$
s(z)=\frac{\Gamma(1 / 2) \Gamma(i z) \zeta(2 i z)}{\Gamma(1 / 2+i z) \zeta(1+2 i z)},
$$

$\zeta$ being the Riemann $\zeta$-function.

Recalling the definition of $P$ described in (3.2), it is clear that $P e$ has finite $G$-norm and hence belongs to $\mathcal{K}$. For our purposes it is more convenient to truncate $e$ in a somewhat different fashion but still bring it into $\mathscr{K}$. We choose

$$
1<a_{0}<b<c<a
$$

and $\xi$ in $C^{\infty}(\mathbf{R})$ so that

$$
\xi(y)= \begin{cases}0 & \text { for } y>c, \\ 1 & \text { for } y<b .\end{cases}
$$

We now define $f$ equal to $e$ except for the zero Fourier coefficient in the region $y>b$ where we set

$$
f^{(0)}(y)=\left\{y^{1 / 2} \xi y^{i z}, y^{3 / 2}\left(\xi y^{i z}\right)^{\prime}\right\}+s(z)\left\{y^{1 / 2-i z}, i z y^{1 / 2-i z}\right\} .
$$

It follows from the above discussion that $f$ belongs to $\mathcal{H}_{G}$ and from (2.16) that $f$ is orthogonal to $\mathscr{Q}_{-}$. Comparing (4.7) and (4.9) shows that

$$
f=e+\left\{y^{1 / 2}(\xi-1) y^{i z}, y^{3 / 2}\left((\xi-1) y^{i z}\right)^{\prime}\right\}
$$

clearly $f$ belongs to $D(A)$ when $\operatorname{Im} z<-1 / 2$. It follows then from (4.5) that

$$
A f=i z f+k
$$

where

$$
k=k^{(0)}=\left\{y^{1 / 2} \xi^{\prime} y^{1+i z}, y^{3 / 2}\left(\xi^{\prime} y^{1+i z}\right)^{\prime}\right\}
$$

Recalling the definition of $\mathscr{Q}_{ \pm}$, it is clear that $k$ is orthogonal to $\mathscr{Q}_{-}$and $\mathscr{D}_{+}$ and so lies in $\mathcal{K}$; one sees by inspection that $k(z)$ is an entire function of $z$.

Next we operate on (4.10) by $P_{+}$; since $k$ lies in $\mathcal{K}, P_{+} k=k$ and hence we get

$$
P_{+} A f=i z P_{+} f+k \text {. }
$$

Set $g=P_{+} f$. Since $f$ is orthogonal to $\mathscr{D}_{-}$and belongs to $D(A)$, the relation (3.7) is applicable. Using (4.12) we obtain

$$
B g=i z g+k \text {. }
$$

For $i z$ in the resolvent set of $B$, the relation (4.13) can be rewritten as

$$
g=-(i z I-B)^{-1} k \text {. }
$$

We conclude from this and Corollary 3.3 that 
LEMMA 4.1. $g(z)$ can be extended to be meromorphic in the whole complex plane, having poles at most at the points -i times the spectrum of $B$, that is $-i \sigma(B)$.

We can now prove

THEOREM 4.2. The Eisenstein series $e$ can be extended to be meromorphic in the whole complex plane with poles at most at the points-io(B); the analytic continuation of $e$ is an eigenfunction of $A$.

REMARK. The analytic continuation of $\mathrm{Pe}(z)$ is in the $\mathcal{H}_{G}$ topology, that of the zero Fourier coefficient of $e$ is pointwise.

Proof. By construction $e(z)=g(z)$ for $\operatorname{Im} z<-1 / 2$ except for the zero Fourier coefficient when $y>b$ and there $e^{(0)}(z)$ is given by (4.7) which holds for $y>a_{0}$. We see from (3.2) that the action of $P_{+}$effects only the zero Fourier coefficient of data and this only for $y>a$. Hence it follows from (4.9)' and (4.8) that the zero Fourier coefficient of $g(z)$ is also given by (4.7) for $a_{0}<y<b$. Thus for $\operatorname{Im} z<-1 / 2, e(w, z)$ can be reconstructed from $g(w, z)$. Now according to Lemma $4.1, g(z)$ has an analytic continuation into $-i$ times the resolvent set of $B$, in symbols $-i \rho(B)$. Since the first term in (4.7) obviously extends analytically into $-i \rho(B)$ so does the second; in particular $s(z)$ can be continued analytically into this region. Finally we note that this implies that the relation (4.5) can also be continued analytically at least in the sense of distributions. It then follows by elliptic regularity theory that the so extended function satisfies (4.5) in the pointwise sense. This completes the proof of Theorem 4.2.

From formula (4.7) we conclude

COROLlary 4.3. The poles of $s(z)$ occur at most at the points-io(B).

5. The translation representations. So far we have managed to avoid the complications which result from the energy form being indefinite; in fact $E$ has played only a minor role in our discussion. We must now confront this problem.

By definition $G=E+2 K$; since $K$ is compact with respect to $G, E$ is positive on a subspace of finite codimension. It is clear from the expression (2.2) that $E$ is nonnegative on the $E$-orthogonal complement of the positive eigenspaces of $L$. Denote the positive eigenfunctions and eigenvalues of $L$ by $\left(q_{j}, \lambda_{j}^{2}\right), j=1, \ldots, m$ with $\lambda_{j}>0$ :

$$
L q_{j}=\lambda_{j}^{2} q_{j}
$$

We set

$$
f_{j}^{ \pm}=\left\{q_{j}, \pm \lambda_{j} q_{j}\right\}
$$

It is easy to verify that $f_{j}^{ \pm}$belongs to $\mathcal{H}_{G}$ and that

$$
\begin{gathered}
A f_{j}^{ \pm}= \pm \lambda_{j} f_{j}^{ \pm} ; \\
E\left(f_{j}^{+}, f_{k}^{+}\right)=0=E\left(f_{j}^{-}, f_{k}^{-}\right) \text {for all } j, k ;
\end{gathered}
$$


and

$$
E\left(f_{j}^{+}, f_{k}^{-}\right)= \begin{cases}0 & \text { for } j \neq k \\ -\lambda_{j}^{2} & \text { for } j=k\end{cases}
$$

We now set

$$
\begin{gathered}
\mathscr{P}_{+}=\operatorname{span} \text { of }\left\{f_{j}^{+}\right\}, \quad \mathscr{P}_{-}=\operatorname{span} \text { of }\left\{f_{j}^{-}\right\}, \\
\mathscr{P}=\mathscr{P}_{+}+\mathscr{P}_{-}
\end{gathered}
$$

and denote the $E$-orthogonal complement of $\mathscr{P}$ in $\mathcal{H}_{G}$ by $\mathcal{H}_{G}^{\prime}$. E is nonnegative on $\mathcal{H}_{G}^{\prime}$, positive if $A$ has no null vectors.

We denote by $Q^{\prime}$ the $E$-orthogonal projection of $\mathcal{H}_{G}$ onto $\mathcal{H}_{G}^{\prime}$; using formulas (5.3) and (5.3)' we can write

$$
Q^{\prime} f=f+\sum a_{j}^{+} f_{j}^{+}+\sum a_{j}^{-} f_{j}^{-}
$$

where

$$
a_{j}^{+}=E\left(f, f_{j}^{-}\right) / \lambda_{j}^{2}, a_{j}^{-}=E\left(f, f_{j}^{+}\right) / \lambda_{j}^{2} .
$$

If $A$ has null vectors, they will span a finite dimensional subspace $\mathscr{G}$ and on the quotient space

$$
\mathcal{H}_{E}^{\prime}=\mathcal{H}_{G}^{\prime} / \mathcal{F}
$$

$E$ is positive definite and equivalent to $G$. Clearly $\mathcal{H}_{E}^{\prime}$ is an invariant space for the operator $U(t)$.

It is easy to show that

$$
\mathscr{D}_{-} \perp \mathscr{P}_{+}, \mathscr{D}_{+} \perp \mathscr{P}_{-} \text {and } \mathscr{P} \cap \mathscr{D}_{-}=\{0\}=\mathscr{P} \cap \mathscr{D}_{+} \text {, }
$$

but it is not true in general that $\mathscr{D}_{-} \perp \mathscr{P}_{-}$or that $\mathscr{D}_{+} \perp \mathscr{P}_{+}$; thus the subspaces $\mathscr{D}_{-}$and $\mathscr{D}_{+}$do not in general lie in $\mathcal{H}_{E}^{\prime}$. The next best thing is to replace $\mathscr{D}_{ \pm}$in our considerations by their $E$-orthogonal projections $\mathscr{D}_{ \pm}^{\prime}$ in $\mathcal{H C}_{E}^{\prime}$; that is by

$$
\mathscr{D}_{ \pm}^{\prime}=Q^{\prime} \mathscr{D}_{ \pm} \text {. }
$$

Strictly speaking one has to mod out $g$ in the right member of $(5.6)^{\prime}$; however since $\mathscr{D}_{ \pm} \cap \mathcal{G}=\{0\}$ this causes no confusion.

The projection operator $Q^{\prime}$ commutes with $U(t)$. It follows from this and properties (2.14) (i) and (ii) that

$$
\begin{aligned}
& \text { (i) } U(t) \mathscr{Q}_{ \pm}^{\prime} \subset \mathscr{Q}_{ \pm}^{\prime} \text { for } t \gtrless 0, \\
& \text { (ii) } \cap U(t) \mathscr{Q}_{-}^{\prime}=\{0\}=\bigcap U(t) \mathscr{Q}_{+}^{\prime} .
\end{aligned}
$$

It can be shown that $\mathscr{D}_{ \pm}^{\prime}$ also satisfy

$$
\overline{U U(t) \mathscr{D}_{-}^{\prime}}=\mathcal{H}_{c}^{\prime}=\overline{\bigcup U(t) \mathscr{D}_{+}^{\prime}},
$$

where $\mathcal{H}_{c}^{\prime}$ is the $E$-orthogonal complement of the eigenfunctions of $A$ in $\mathcal{H}_{E}^{\prime}$. For the proof of this property we refer to Corollary 6.14 of our monograph [6]; the main ingredient of the proof is Theorem 2.1.

According to the translation representation theorem (see Chapter 2 of [6]), any subspace satisfying (i)_-(iii)_ (or (i) $\left.)_{+}-(\mathrm{iii})_{+}\right)$of $(5.7)$ can be used to 
construct a translation representation of $\mathcal{H}_{c}^{\prime}$, i.e. a unitary map $T_{-}$(or $T_{+}$) of $\mathcal{H}_{c}^{\prime}$ onto a vector valued space of functions of the form $L_{2}(\mathbf{R}, \Re)$, $\Re$ being an auxiliary Hilbert space, with the properties:

$$
\begin{aligned}
& T_{ \pm}: f \in \mathcal{H}_{c}^{\prime} \rightarrow k_{ \pm} \in L_{2}(\mathbf{R}, \mathcal{T}), \\
& (\alpha) \quad E(f)=\int\left\|k_{ \pm}\right\|_{\Re}^{2} d s, \\
& (\beta) \quad U(t) f \rightarrow k_{ \pm}(s-t), \\
& (\gamma) \quad T_{ \pm} \text {maps } \mathscr{D}_{ \pm}^{\prime} \text { onto } L_{2}\left(\mathbf{R}_{ \pm}, \Re\right) .
\end{aligned}
$$

We need not rely on this general existence theorem for we can, as we shall show below, obtain explicit formulas for these primed translation representations. We treat only the $\mathscr{D}_{+}^{\prime}$-representation but it will be clear that the OD'--representation can be treated analogously. We begin by constructing a translation representation for the elements of $U(t) \mathscr{D}_{+}$. Given automorphic data $f=\left(f_{1}, f_{2}\right)$ defined on all $\Pi$ with locally finite $G$-norm, denote, as in (2.15), by $f_{i}^{(0)}$ the zero Fourier coefficients of $f_{i}, i=1,2$ :

$$
f_{i}^{(0)}(y)=\int_{-1 / 2}^{1 / 2} f_{i}(x, y) d x, \quad 0<y<\infty .
$$

We define the translation representer of $f$ to be

$$
T_{+} f=\frac{1}{\sqrt{2}}\left[\partial_{s}\left(e^{-s / 2} f_{1}^{(0)}\left(e^{s}\right)\right)-e^{-s / 2} f_{2}^{(0)}\left(e^{s}\right)\right],-\infty<s<\infty .(5.10)_{+}
$$

It is easy to see that $T_{+}$is a linear mapping, continuous from $\mathcal{H}_{G}$ into $L_{2}^{\text {loc }}(\mathbf{R})$.

LEMMA 5.1. $T_{+}$commutes with translations:

$$
T_{+} U(t) f=T(t) T_{+} f
$$

here $T(t)$ denotes translation to the right by $t$ units.

Proof. Let $u(x, y, t)$ be the solution to the non-Euclidean wave equation with automorphic initial data $f$ :

$$
u_{t t}=L u ; \quad u(0)=f_{1} \text { and } u_{t}(0)=f_{2} \text {. }
$$

Then for each time $t, u$ will be automorphic and in particular it will be periodic in $x$ of period 1 for all $y>0$. The zero Fourier coefficient $u^{(0)}(y, t)$ will therefore satisfy the equation

$$
u_{t t}^{(0)}=y^{2} u_{y y}^{(0)}+u^{(0)} / 4 \text { for all } y>0 .
$$

As we have seen in $\$ 2$, the change of variables

$$
s=\log y, \quad v=u^{(0)} / \sqrt{y}
$$

transforms (5.12) into the classical wave equation:

$$
v_{t t}=v_{s s}
$$

the initial data goes over into

$$
v(0)=e^{-s / 2} f_{1}^{(0)}\left(e^{s}\right) \text { and } v_{t}(0)=e^{-s / 2} f_{2}^{(0)}\left(e^{s}\right) .
$$


Setting

$$
k(s, t)=v_{s}-v_{t},
$$

it follows from (5.10) and (5.15) that

$$
k(s, 0)=\sqrt{2} T_{+} f
$$

Since the solution data at time $t$ is $U(t) f$, we have similarly

$$
k(s, t)=\sqrt{2} T_{+} U(t) f .
$$

Combining (5.14) and (5.16) we see that

$$
\partial_{t} k+\partial_{s} k=0
$$

and hence that

$$
k(s, t)=k(s-t, 0) .
$$

Finally on combining (5.18) with (5.17) we obtain the assertion of the lemma.

Note that in the proof of Lemma 5.1 we used only the fact that $f$ was periodic in $x$ with period 1 . We therefore have

COROllary 5.2 Let $f$ be data periodic in $x$ with period 1 and with locally finite $G$-norm. Define $T_{+}$as before by (5.9) and (5.10) $)_{+}$. Then Lemma 5.1 holds for $f$.

Next we consider data $d_{0}$ defined as in (2.13):

$$
d_{0}=\left\{y^{1 / 2} \varphi(y),-y^{3 / 2} \varphi^{\prime}(y)\right\}
$$

where $\varphi$ lies in $C_{0}^{\infty}(\mathbf{R})$ and vanishes for $y<a$. Since $d_{0}$ is periodic in $x$, the corresponding data $d$ obtained by summing its $\gamma$-translates over the right cosets $\Gamma_{\infty} \backslash \Gamma$ is automorphic:

$$
d(w)=\sum_{\Gamma_{\infty} \backslash \Gamma} d_{0}(\gamma w) .
$$

Since $\varphi=0$ for $y<a, d_{0}(w)=0$ for $y<a$; so $d_{0}(\gamma w)=0$ for $w$ in $F$ and $\gamma \notin \Gamma_{\infty}$ and therefore $d=d_{0}$ on the fundamental domain $F$; hence (5.19) shows that $d$ belongs to $\mathscr{D}_{+}$. As we showed in $\$ 2$, the solution of the non-Euclidean wave equation with initial data $d_{0}$ of the form (5.19) is given by

$$
u_{0}(w, t)=y^{1 / 2} \varphi\left(y e^{-t}\right) .
$$

Clearly $u_{0}(w, t)$ is periodic in $x$. The automorphic solution of the nonEuclidean wave equation with initial data $d$ given by (5.20) is

$$
u(w, t)=\sum_{\Gamma_{\infty} \backslash \Gamma} u_{0}(\gamma w, t) .
$$

The reasoning used above shows that $u(w, t)=u_{0}(w, t)$ for $w$ in $F$ and $t \geq 0$.

LEMMA 5.3. For $d_{0}$ and $d$ given by (5.19) and (5.20), respectively,

(a) $T_{+} d_{0}=T_{+} d$;

(b) $E_{F}(d)=\int_{-\infty}^{\infty}\left|T_{+} d\right|^{2} d s$. 
Proof. Since $u=u_{0}$ in $F$ for $t \geqslant 0$, we have

$$
T_{+}(U(t) d)=T_{+}\left(U(t) d_{0}\right) \text { for } t \geqslant 0 \text { and } s>\log a \text {. }
$$

Using this relation and (5.11) of Lemma 5.1 and its corollary, we conclude that

$$
T(t) T_{+} d=T(t) T_{+} d_{0} \text { for } t \geqslant 0 \text { and } s>\log a .
$$

Since this holds for all $t \geqslant 0$, it follows that it must hold for all $s$. This proves part (a).

Substituting (5.19) into the definition $(5.10)_{+}$of $T_{+}$we obtain

$$
T_{+} d_{0}=\sqrt{2} e^{s} \varphi^{\prime}\left(e^{s}\right)
$$

The proof of part (b) is now immediate since by definition (2.2)"

$$
\begin{aligned}
E_{F}(d) & =\iint_{F}\left\{y\left|\partial_{y} \varphi\right|^{2}+\frac{\left|y^{3 / 2} \varphi^{\prime}(y)\right|^{2}}{y^{2}}\right\} d x d y \\
& =2 \int_{0}^{\infty} y\left|\varphi^{\prime}\right|^{2} d y=2 \int_{-\infty}^{\infty} e^{2 s}\left|\varphi^{\prime}\left(e^{s}\right)\right|^{2} d s
\end{aligned}
$$

which by (5.22)

$$
=\int_{-\infty}^{\infty}\left|T_{+} d_{0}\right|^{2} d s
$$

Combining this with the assertion of part (a) gives part (b). This completes the proof of the lemma.

Combining Lemma 5.3 with Lemma 5.1 and its corollary, we deduce the following

Corollary 5.4. (a) $T_{+}\left(U(t) d_{0}\right)=T_{+}(U(t) d)$;

(b) $E_{F}(U(t) d)=\int_{-\infty}^{\infty}\left|T_{+} d\right|^{2} d s$.

We are now ready to construct the $\mathscr{D}_{+}^{\prime}$-translation representaion. Recall that $\mathscr{D}_{+}^{\prime}=Q^{\prime} \mathscr{Q}_{+}$; that is the elements $d_{+}^{\prime}$ of $\mathscr{D}_{+}^{\prime}$ are obtained by projecting the elements $d_{+}$of $\mathscr{D}_{+}$into $\mathcal{H}_{E}^{\prime}$ :

$$
d_{+}^{\prime}=d_{+}+p_{-}+p_{+},
$$

where $p_{ \pm}$belongs to $\mathscr{P}_{ \pm}$. According to (5.6), $\mathscr{D}_{+}$is orthogonal to $\mathscr{P}_{-}$and it follows from this and the relations (5.5) and (5.5) that $p_{+}=0$ :

$$
d_{+}^{\prime}=d_{+}+p_{-} .
$$

Using (5.3) and (5.3)' we deduce that

$$
E_{F}\left(d_{+}\right)=E_{F}\left(d_{+}^{\prime}\right) \text {. }
$$

This shows that $Q^{\prime}$ defines an isometric map on $\mathscr{Q}_{+}$to $\mathscr{Q}_{+}^{\prime}$. It follows that the translation representer of $d_{+}^{\prime}$ can be obtained directly by means of the operator $T_{+}$as we now prove:

LEMMA 5.5. $T_{+} d_{+}^{\prime}=T_{+} d_{+}$. 
Proof. Since $d_{+}$and $d_{+}^{\prime}$ differ only by an element of $\mathscr{P}_{-}$and since $\mathscr{P}_{-}$is spanned by the $\left\{f_{j}^{-}\right\}$of (5.2), it suffices to show that

$$
T_{+} f_{j}^{-}=0 \text {. }
$$

We note that $U(t) f_{j}^{-}=e^{-\lambda{ }^{t}} f_{j}^{-}$. Using this fact and Lemma 5.1 we conclude that

$$
T_{+} f_{j}^{-}=\text {const } e^{\lambda_{s} s} .
$$

As noted in (5.6), $\mathscr{P}_{-}$is orthogonal to $\mathscr{D}_{+}$; we now show that $T_{+} f=0$ for $s>\log a$ for any element $f$ in $\mathscr{H}_{G}$ which is orthogonal to $\mathscr{D}_{+}$. To see this take $d$ in $\mathscr{D}_{+}$of the form (5.20); the restriction of $d$ to $F$ is given by (5.19). Hence we have

$$
0=E_{F}(f, d)=\iint_{F}\left\{y\left(\partial_{y} \frac{f_{1}}{\sqrt{y}}\right) \overline{\varphi^{\prime}}-\frac{f_{2} y^{3 / 2} \overline{\varphi^{\prime}}}{y^{2}}\right\} d x d y .
$$

Performing that $x$ integration, we can replace $f_{1}$ and $f_{2}$ by their zero Fourier coefficients $f_{1}^{(0)}$ and $f_{2}^{(0)}$; changing the other variable of integration $y$ into $s=\log y$, the above equation becomes

$$
0=\int_{-\infty}^{\infty}\left\{\partial_{s}\left(e^{-s / 2} \hat{f}_{1}\right)-e^{-s / 2} \hat{f}_{2}\right\} \partial_{s} \overline{\varphi\left(e^{s}\right)} d s
$$

Recalling the definition $(5.10)_{+}$of $T_{+}$we see that this relation says that $T_{+} f$ is $L_{2}$-orthogonal to $\partial_{s} \varphi\left(e^{s}\right)$. Since such functions are dense in $L_{2}\left(\mathbf{R}_{+}+\log a\right)$, we can conclude that $T_{+} f=0$ for $s>\log a$. Comparing this with (5.27) we see that $T_{+} f_{j}^{-} \equiv 0$.

LEMMA 5.6. (a) Let $p$ be an eigenfunction of $L$ with negative eigenvalue $-\nu^{2}$. Denote by $g_{ \pm}=\{p, \pm i v p\}$ the corresponding eigenfunctions of $A$. Then

$$
T_{+}\left(g_{ \pm}\right)=0 \text {. }
$$

(b) Let $f$ be an arbitrary automorphic data in $\mathcal{H}_{E}^{\prime}$ and denote by $f_{c}^{\prime}$ the projection of $f$ into $\mathcal{H}_{c}^{\prime}$. Then

$$
T_{+} f=T_{+} f_{c}^{\prime}
$$

Proof. The proof of (a) is entirely analogous to that of (5.26) since $g_{ \pm}$ belongs to the point spectrum of $A$ in $\mathcal{H}_{E}^{\prime}$ and because of this can easily be shown to be orthogonal to $\mathscr{D}_{ \pm}^{\prime}$ and hence to be orthogonal to $\mathscr{D}_{ \pm}$. Part (b) follows from part (a) since $f-f_{c}^{\prime}$ can be expanded as a convergent series in eigenfunctions of the kind considered in part (a). Recalling that $T_{+}$is continuous from $\mathcal{H}_{G}$ to $L_{2}^{\text {loc }}$ and that the $G$ and $E$ norms are equivalent on $\mathcal{H}_{E}^{\prime}$, assertion (b) now follows.

THEOREM 5.7. $T_{+}$is the $\mathscr{Q}_{+}^{\prime}$-translation representation of $\mathcal{H}_{c}^{\prime}$.

Proof. We shall verify properties (5.8) where auxiliary Hilbert space $\mathscr{T}$ is C: By Lemma 5.1, $T_{+}$is a translation representation and hence satisfies property $(\beta)$. By part (b) of Lemma $5.3, T_{+}$is isometric on $\mathscr{Q}_{+}$. Formula (5.22) shows that $T_{+}$maps $\mathscr{Q}_{+}$onto $L_{2}(R+\log a, C)$. Since by Lemma 5.5, $T_{+} d_{+}^{\prime}=T_{+} d_{+}$and by $(5.25) E_{F}\left(d_{+}^{\prime}\right)=E_{F}\left(d_{+}\right)$, it follows that $T_{+}$maps $\mathscr{D}_{+}^{\prime}$ 
isometrically onto $L_{2}(\mathbf{R}+\log a, \mathbf{C})$. This proves property $(\gamma)$. Since by property (iii) of (5.7) the translates of $\mathscr{Q}_{+}^{\prime}$ by $U(t)$ span $\mathcal{F C}_{c}^{\prime}$ and since the isometry persists under such translations, it follows that $T_{+}$is unitary and hence is an outgoing translation representation of $\mathcal{H C}_{c}^{\prime}$ with respect to $\mathscr{D}_{+}^{\prime}$.

The analogue of Theorem 5.7 holds for the $\mathscr{D}_{-}^{\prime}$-translation representation of $\mathcal{H C}_{c}^{\prime}$ with $T_{+}$replaced by

$$
T_{-} f=-\frac{1}{\sqrt{2}}\left[\partial_{s}\left(e^{s / 2} f_{1}^{(0)}\left(e^{-s}\right)\right)-e^{s / 2} f_{2}^{(0)}\left(e^{-s}\right)\right] .
$$

Formulas $(5.10)_{ \pm}$are the explicit translation representation formulas alluded to in the introduction. We recall that for the Euclidean wave equation, the translation representation is given in terms of the Radon transform of the data defined by integrals along all straight lines in the plane. The integrals in (5.8) are over a one-parameter set of curves in $F$ that are the images of the horocycles through infinity under the projection of the Poincaré plane $\Pi$ onto $F$.

The inversion of $T_{+}$can be accomplished in three steps. Let $k(s)=T_{+} f$ and define $\varphi$ by

$$
\varphi(y)=\int_{-\infty}^{\log y} k(s) d s
$$

(i) Set

$$
g_{0}(w)=\left\{y^{1 / 2} \varphi(y),-y^{3 / 2} \varphi^{\prime}(y)\right\} .
$$

(ii) Define

$$
g(w)=\sum_{\Gamma_{\infty} \backslash \Gamma} g_{0}(\gamma w)
$$

(iii) Project $g$ into $\mathcal{H}_{G}^{\prime}$ :

$$
f=Q^{\prime} g .
$$

Proof. Suppose $f$ belongs to $\mathscr{D}_{+}^{\prime}$. Then by definition there exists a $d_{+}$in $\mathscr{D}_{+}$such that $Q^{\prime} d_{+}=d_{+}^{\prime}=f$. It is clear from (5.19), (5.20) and (5.22) that $g$, defined as above, is this $d_{+}$; this proves (5.32) when $f \in \mathscr{D}_{+}^{\prime}$. Next suppose that $f=U(t) d_{+}$; then, since $Q^{\prime}$ commutes with $U(t), f=Q^{\prime} U(t) d_{+}$. Comparing the relations (5.19) through (5.20), with (5.29) to (5.31) we conclude that $g=U(t) d_{+}$; the relation (5.32) therefore holds in this case as well. Finally since $\cup U(t) \mathscr{D}_{+}^{\prime}$ is dense in $\mathcal{H}_{c}^{\prime}$, it follows by continuity that (5.32) holds for all $f$ in $\mathcal{H}_{c}^{\prime}$.

Using Lemma 5.6, we see that formula (5.32) can be used to project any $f$ in $\mathcal{H}_{E}^{\prime}$ into $\mathcal{H C}_{c}^{\prime}$.

We conclude this section by defining the scattering operator $S^{\prime}$ as the mapping of the $\mathscr{D}_{-}^{\prime}$-representation onto the $\mathscr{D}_{+}^{\prime}$-representation:

$$
S^{\prime}: T_{-} f \rightarrow T_{+} f \text { for } f \text { in } \mathcal{H}_{c}^{\prime} \text {. }
$$

6. The spectral representation. A representation in which the action of $U(t)$ is multiplication by exp (iot) is called a spectral representation. It is clear that the Fourier transform of a translation representation satisfies this criterion. In this section we shall study the spectral representation of $U$, restricted to $\mathcal{H}_{c}^{\prime}$, 
so obtained from the $\mathscr{T}_{+}^{\prime}$ and $\mathscr{D}_{-}^{\prime}$ translation representation treated in $\$ 5$ :

$$
\left(\mathscr{T}_{ \pm} f\right)(\sigma)=\frac{1}{\sqrt{2 \pi}} \int_{-\infty}^{\infty} e^{i \sigma s}\left(T_{+} f\right)(s) d s
$$

We now prove

THEOREM 6.1. The $\mathscr{D}_{ \pm}^{\prime}$-spectral representation can be expressed as

$$
\mathcal{T}_{ \pm} f(\sigma)=\frac{1}{\sqrt{2 \pi}} E_{F}\left(f, \overline{e_{ \pm}(\sigma)}\right)
$$

where $e_{ \pm}(z), z=\sigma+i \tau$, are analytic continuations of the Eisenstein series generated by

$$
h_{ \pm}(w, z)=\frac{ \pm 1}{\sqrt{2} i z}\left\{y^{1 / 2 \pm i z},-i z y^{1 / 2 \pm i z}\right\} .
$$

REMARK. It should be noted that $h_{+}$and $h_{-}$are closely related to but somewhat different than $h$ in (4.1). In particular

$$
h_{-}(z)=-h(-z) / \sqrt{2} i z
$$

since the Eisenstein series $e$ corresponding to $h$ converges for $\operatorname{Im} z<-1 / 2$, the Eisenstein series $e_{-}(z)$ converges for $\operatorname{Im} z>1 / 2$, and the zero Fourier coefficient of $e_{-}$is related to that of $e$ by

$$
e_{-}^{(0)}(z)=\frac{-1}{\sqrt{2} i z} e^{(0)}(-z) .
$$

An explicit formula for $e^{(0)}$ is given in (4.7). On the other hand $h_{+}(z)$ is essentially the same as $(\sqrt{2} i z)^{-1} h(z)$ except that its second component has the opposite sign. This sign change has no effect on the convergence of the corresponding Eisenstein series $e_{+}(z)$ nor on its analytic extension. The analogue of (4.7) is now

$$
\sqrt{2} i z e_{+}^{(0)}(z)=\left\{y^{1 / 2+i z},-i z y^{1 / 2+i z}\right\}+s(z)\left\{y^{1 / 2-i z},-i z y^{1 / 2-i z}\right\} .
$$

Proof. We shall treat only the $\mathscr{D}_{+}^{\prime}$-representation. Since $\mathscr{T}_{+}$is the composite of the two unitary maps $T_{+}$and the Fourier transform, it is itself unitary. Thus $\mathcal{T}_{+}$is continuous; so it suffices to prove (6.2) for a dense subset of $\mathcal{F K}_{c}^{\prime}$; for our purpose this will be the set of data $f$ for which $T_{+} f$ is smooth with compact support, and for which

$$
\int T_{+} f(s) d s=0
$$

We shall derive bounds for such $f$ by inverting $T_{+}$; see the end of $\S 5$. Set

$$
\varphi(y)=\frac{1}{\sqrt{2}} \int_{-\infty}^{\log y} T_{+} f(s) d s
$$

and

$$
g_{0}(y)=\left\{y^{1 / 2} \varphi(y),-y^{3 / 2} \varphi^{\prime}(y)\right\} .
$$

Then $g_{0}(y)$ has compact support in $y$; as a consequence only a finite number 
of images $\gamma^{-1} F$ of $F$ lie in that portion of the support of $g_{0}$ which is contained in the strip

$$
S=\{-1 / 2<x<1 / 2, y>0\}
$$

Thus the sum

$$
g(w)=\sum_{\Gamma_{\infty} \backslash \Gamma} g_{0}(\gamma w)
$$

has only a finite number of nonvanishing terms on $F$ and is of compact support in $F . g$ is automorphic and, as shown at the end of $\S 5$,

$$
f=Q^{\prime} g \text {, }
$$

and by (5.26)

$$
T_{+} f=T_{+} g
$$

By definition (5.5) of $Q^{\prime}$,

$$
f-g \in \mathscr{P} \text {. }
$$

N.b. It is easy to show that $f-g$ actually belongs to $\mathscr{P}_{-}$, but the weaker result (6.7) suffices, here.

Substituting (6.6) into (6.1) we get

$$
\mathcal{T}_{+} f=\frac{1}{\sqrt{2 \pi}} \int_{-\infty}^{\infty} e^{i \sigma s}(T+g)(s) d s .
$$

Recalling formula (5.10) + defining $T_{+}$and making the change of variable: $s \rightarrow \log y$, the relation (6.8) becomes

$$
\mathscr{T}_{+} f=\frac{1}{2 \sqrt{\pi}} \int_{0}^{\infty}\left[\int_{-1 / 2}^{1 / 2} y^{i \sigma}\left\{y \partial_{y}\left(\frac{g_{1}(x, y)}{\sqrt{y}}\right)-\frac{g_{2}(x, y)}{\sqrt{y}}\right\} d x\right] \frac{d y}{y} .
$$

The remainder of the proof of Theorem 6.1 splits into three parts:

Step 1. Continue (6.8)' analytically into the half plane $\operatorname{Im} z<-1 / 2$ and then rewrite it as

$$
\mathcal{T}_{+} f=\frac{1}{\sqrt{2 \pi}} E_{S}\left(g, \overline{h_{+}(z)}\right)
$$

here $E_{S}$ denotes the invariant form of the energy integral given in (2.2) but integrated over the strip $S$ instead of $F$.

Step 2. Replace the integral over the strip by a sum of integrals over all the $\gamma^{-1} F$ 's contained in $S$. Using the fact that $g$ is automorphic this gives

$$
\begin{aligned}
\mathscr{T}_{+} f & =\frac{1}{\sqrt{2 \pi}} \sum_{\Gamma_{\infty} \backslash \Gamma} E_{\gamma^{-1} F}\left(g, \overline{h_{+}(z)}\right) \\
& =\frac{1}{\sqrt{2 \pi}} \sum_{\Gamma_{\infty} \backslash \Gamma} E_{F}\left(g, \overline{h_{+}(\gamma w, z)}\right) \\
& =\frac{1}{\sqrt{2 \pi}} E_{F}\left(g, \sum_{\Gamma_{\infty} \backslash \Gamma} \overline{h_{+}(\gamma w, z)}\right)=E_{F}\left(g, \overline{e_{+}(z)}\right) .
\end{aligned}
$$

Note that the sum defining the Eisenstein series converges for $\operatorname{Im} z<-1 / 2$ 
and that $e_{+}(z)$ can be continued analytically back to real $z$.

Step 3. Show for the eigenfunctions $\left\{f_{j}^{ \pm}\right\}$that

$$
E_{F}\left(f_{j}^{ \pm}, \bar{e}_{+}(\sigma)\right)=0 .
$$

Since the $f_{j}^{ \pm}$span $\mathscr{P}$ and since by (6.7) $f-g \in \mathcal{P}$, the relation (6.2) is an immediate consequence of (6.10) and (6.11).

Proof of STEP 1. Since $T_{+} g(s)$ has compact support, it is clear that $\mathcal{T}_{+} f$ as defined by (6.8), and hence (6.8)', is entire in $\sigma$ and can be continued analytically into the half-plane $\operatorname{Im} z<-1 / 2, z=\sigma+i \tau$. Notice that the support for the individual terms in (6.8) are bounded away from $y=\infty$ but not from $y=0$. It follows that each of these terms is integrable for large $y$; for small $y$ the factor $y^{i z}$ decays fast enough if $\operatorname{Im} z<-1 / 2$, and we now make use of this fact to show that (6.8)' exists as a double integral.

As noted before, all but a finite number of the terms in (6.5) vanish on $F$. Thus $g(w)$ is bounded on $F$ and since $g$ is automorphic it is also bounded on $S$. It follows that for $\operatorname{Im} z<-1 / 2$ the second term on the right in $(6.8)^{\prime}$ is integrable near $y=0$. The first term can be written as

$$
y \partial_{y}\left(\frac{g_{1}}{\sqrt{y}}\right)=\sqrt{y} \partial_{y} g_{1}-\frac{g_{1}}{2 \sqrt{y}} ;
$$

the same reasoning as above shows that the contribution of the second term on the right in this expression to (6.8)' is also integrable for small $y$. Thus it only remains to consider the integrability of

$$
y^{i z-1 / 2} \partial_{y} g_{1}
$$

Recall that by (6.5)

$$
g=\sum_{\Gamma_{\infty} \backslash \Gamma} g_{0}(\gamma w)
$$

where $g_{0}$ is a smooth function of $y$ alone with compact support, say in the interval $(m, M)$. Hence

$$
g_{0}(\gamma w)=g_{0}(v)
$$

where

$$
u+i v=\gamma w=\frac{a w+b}{c w+d} \quad \text { and } \quad v=\frac{y}{|c w+d|^{2}}
$$

Now

$$
\frac{\partial g_{0}(\gamma w)}{\partial y}=\frac{\partial g_{0}(v)}{\partial v} \cdot \frac{\partial v}{\partial y} \quad \text { and } \quad\left|\frac{\partial v}{\partial y}\right| \leqslant \frac{1}{|c w+d|^{2}}=\frac{v}{y} .
$$

It follows that

$$
\left|\frac{\partial g_{0}(\gamma w)}{\partial y}\right| \leqslant \text { const } \frac{M}{y} \quad \text { for all } \gamma .
$$

Since the number of terms in (6.5) which do not vanish on any transform $\gamma F$ of $F$ is finite and independent of $\gamma$, we conclude that 


$$
\left|\frac{\partial g_{1}}{\partial y}\right| \leqslant \text { const } \frac{M}{y} .
$$

This estimate is sufficient to assure the integrability of (6.12) over the strip $S$ when $\operatorname{Im} z<-1 / 2$.

We may now proceed formally to bring $(6.8)^{\prime}$ into the desired invariant form. We write

$$
\iint_{S} y^{i z} \partial_{y}\left(\frac{g_{1}}{\sqrt{y}}\right) d x d y=\iint_{S}\left\{y^{i z-1 / 2} \partial_{y} g_{1}-\frac{1}{2} y^{i z-3 / 2} g_{1}\right\} d x d y
$$

splitting the first term, this can be rewritten as

$$
=\iint_{S}\left\{\frac{i z+1 / 2}{i z} y^{i z-1 / 2} \partial_{y} g_{1}-\frac{1}{2 i z} y^{i z-1 / 2} \partial_{y} g_{1}-\frac{1}{2} y^{i z-3 / 2} g_{1}\right\} d x d y ;
$$

and integrating the middle term by parts gives

$$
\int_{0}^{\infty} \frac{1}{2 i z} y^{i z-1 / 2} \partial_{y} g_{1} d y=\left.\frac{1}{2 i z} y^{i z-1 / 2} g_{1}\right|_{0} ^{\infty}-\int_{0}^{\infty} \frac{i z-1 / 2}{2 i z} y^{i z-3 / 2} g_{1} d y .
$$

The integrated term vanishes; substituting the remaining term back into (6.13) we get

$$
\iint_{S} y^{i z} \partial_{y}\left(\frac{g_{1}}{\sqrt{y}}\right) d x d y=\frac{1}{i z} \iint_{S}\left\{\partial_{y} g_{1} \cdot \partial_{y} y^{i z+1 / 2}-\frac{g_{1} \cdot y^{i z+1 / 2}}{4 y^{2}}\right\} d x d y .
$$

Note that this integration by parts is the reverse of the one that brought (2.2) into the form (2.2)". The right member of the above equation is in invariant form as can be seen from (1.5) and (1.6). Substituting this back in (6.8) ${ }^{\prime}$ and making use of the definition of $h_{+}$as given in (6.3) we obtain (6.9).

Proof of STEP 2. In order to justify the interchange in the order of summation and integration occurring in (6.10), it suffices to recall the fact, noted in $\$ 4$, that the Eisenstein series converges in $\mathcal{H}_{G}$ when $\operatorname{Im} z<-1 / 2$, except for the first term $h_{+}(w)$. However $h_{+}(w)$ is locally in $\mathcal{H}_{G}$ and since $g$ is of compact support in $F$, even this term causes no difficulty.

According to Theorem 4.2, the Eisenstein function $e_{+}(z)$ is meromorphic in the local $\mathcal{K}_{G}$ topology. Hence if we again make use of the fact that $g$ has compact support in $F$, we see that $E_{F}\left(g, \overline{e_{+}(z)}\right)$ is meromorphic and can be continued analytically back to the real axis. It follows that

$$
\sigma_{+} f(\sigma)=\frac{1}{\sqrt{2 \pi}} E_{F}\left(g, \overline{e_{+}(\sigma)}\right) .
$$

Proof of Step 3. This proof is based on the observation that both $f_{j}^{ \pm}$and $e_{+}(\sigma)$ are eigenfunctions of the generator $A$ and that the standard proof for the orthogonality of eigenfunctions of a skew-Hermitian partial differential operator can be applied in this case even though one of the eigenfunctions is a generalized one. As defined in (5.1)

$$
f_{j}^{ \pm}=\left\{q_{j}, \pm \lambda_{j} q_{j}\right\}
$$

where $q_{j}$ is an eigenfunction of $L: L q_{j}=\lambda_{j}^{2} q_{j}$, belonging to $L_{2}(F)$. The zero 
Fourier coefficient of $q_{j}$ satisfies the ordinary differential equation

$$
y^{2} \frac{d^{2}}{d y^{2}} q_{j}^{(0)}+\frac{1}{4} q_{j}^{(0)}=\lambda_{j}^{2} q_{j}^{(0)}
$$

and hence is of the form

$$
q_{j}^{(0)}=c y^{1 / 2+\lambda}+d y^{1 / 2-\lambda} \text { for } y>a .
$$

Since $q_{j}$ is square integrable on $F$, the coefficient $c$ must be zero. On the other hand $e_{+}(\sigma)$ is in $\mathcal{H}_{G}$ except for its zero Fourier coefficient which by (4.7) is of the form

$$
\frac{1}{\sqrt{2} i z}\left[\left\{y^{1 / 2+i \sigma},-i \sigma y^{1 / 2+i \sigma}\right\}+s(\sigma)\left\{y^{1 / 2-i \sigma},-i \sigma y^{1 / 2-i \sigma}\right\}\right] .
$$

It follows that the $E_{F}$ inner product of $f_{j}^{ \pm}$and $\overline{e_{+}(\sigma)}$ is integrable and that integration by parts is permissible. This gives

$$
\begin{aligned}
i \sigma E_{F}\left(f_{j}^{ \pm}, \overline{e_{+}(\sigma)}\right) & =E_{F}\left(f_{j}^{ \pm}, \overline{A e_{+}(\sigma)}\right) \\
& =-E_{F}\left(A f_{j}^{ \pm}, \overline{e_{+}(\sigma)}\right)=\mp \lambda_{j} E_{F}\left(f_{j}^{ \pm}, \overline{e_{+}(\sigma)}\right)
\end{aligned}
$$

from which (6.11) follows. This completes the proof of Theorem 6.1.

In analogy with Lemma 5.6 we have

LEMMA 6.2. Let $f$ be an arbitrary automorphic data in $\mathcal{H}_{E}^{\prime}$ and denote by $f_{c}^{\prime}$ the projection of $f$ into $\mathcal{H}_{c}^{\prime}$. Then

$$
E_{F}\left(f, \overline{e_{+}(\sigma)}\right)=E_{F}\left(f_{c}^{\prime}, \overline{e_{+}(\sigma)}\right) .
$$

Since $f$ differs from $f_{c}^{\prime}$ by a sum of genuine eigenvectors of $A$, this follows by essentially the same argument as that used in (6.14).

REMARK 6.3. It is easy to see that $\boldsymbol{A}$ has only a discrete set of purely imaginary point eigenvalues. In fact since the zero Fourier coefficient of an associated eigenfunction is of the form

$$
c\left\{y^{1 / 2+i \sigma}, i \sigma y^{1 / 2+i \sigma}\right\}+d\left\{y^{1 / 2-i \sigma}, i \sigma y^{1 / 2-i \sigma}\right\}
$$

and since a point eigenfunction is by definition of finite $G$-norm, both $c$ and $d$ must be zero. It then follows by (2.17) that any such eigenfunction belongs to the subspace $\mathcal{K}$; the assertion is therefore an immediate consequence of Corollary 2.2.

We conclude this section by obtaining the spectral representer for the scattering operator $S^{\prime}$ defined in (5.33). To this end we prove

Lemma 6.4.

$$
e_{+}(\sigma)=-s(\sigma) e_{-}(\sigma) \text {. }
$$

Proof. It is readily verified that $h_{ \pm}$satisfy locally

$$
A h_{ \pm}=-i z h_{ \pm} \text {. }
$$

It follows, along the lines indicated at the beginning of $\S 4$, that $e_{ \pm}$also satisfy locally

$$
A e_{ \pm}=-i z e_{ \pm}
$$


It follows from (6.4)+ that

$$
\sqrt{2} i z e_{+}^{(0)}(z)=\left\{y^{1 / 2+i z},-i z y^{1 / 2+i z}\right\}+s(z)\left\{y^{1 / 2-i z},-i z y^{1 / 2-i z}\right\}
$$

while according to (6.4) _ and (4.7)

$$
-\sqrt{2} i z e_{-}^{(0)}(z)=\left\{y^{1 / 2-i z},-i z y^{1 / 2-i z}\right\}+s(-z)\left\{y^{1 / 2+i z},-i z y^{1 / 2+i z}\right\} .
$$

Therefore

$$
f=e_{+}(z)+s(z) e_{-}(z)
$$

satisfies

$$
f^{(0)}(z)=\text { const }\left\{y^{1 / 2+i z},-i z y^{1 / 2+i z}\right\} \text {. }
$$

Thus for $\operatorname{Im} z>0, f^{(0)}(z)$ belongs to $\mathcal{H}_{G}$. According to Lemma 4.1, $e_{ \pm}(z)-$ $e_{ \pm}^{(0)}(\mathrm{z})$, belong to $\mathcal{H}_{G}$ except when $z$ lies in $-i \sigma(B)$; it follows that for all such $z$ with $\operatorname{Im} z>0, f(z)$ belongs to $\mathcal{H}_{G}$. Since $f$ is a linear combination of $e_{+}$and $\boldsymbol{e}_{-}$, it satisfies

$$
A f=-i z f
$$

Now $A$ has only a finite set of nonimaginary eigenvalues. These are the values $\left\{ \pm \lambda_{j}\right\}$ appearing in (5.1)' which correspond to the positive eigenpairs of $L$. In fact, as explained in $\S 5$, in the quotient space $\mathcal{F}_{E}^{\prime}=\mathcal{F}_{G}^{\prime} / \mathcal{G}$, where $\mathcal{g}$ is the null space of $A, E$ is positive definite and equivalent to $G$. Since $A$ restricted to $\mathcal{H}_{E}^{\prime}$ is skew-Hermitian, it has no nonimaginary eigenvalues on $\mathcal{H}_{E}^{\prime}$. Thus for $\operatorname{Im} z>0$ and $z$ not in $\left\{i \lambda_{j}\right\}$, it follows from the above that $f(z) \equiv 0$. By analytic continuation this holds for real $z$ as well, as asserted in Lemma 6.4.

THEOREM 6.5. The spectral representer of the scattering operator:

$$
\mathfrak{S}^{\prime}: \mathcal{T}_{-} f \rightarrow \mathcal{T}_{+} f
$$

is the multiplicative operator:

$$
\delta^{\prime}(\sigma)=-s(\sigma) .
$$

Proof. It follows from Theorem 6.1 and Lemma 6.4 that

$$
\mathscr{T}_{+} f=E_{F}\left(f, \overline{e_{+}(\sigma)}\right)=-s(\sigma) E_{F}\left(f, \overline{e_{-}(\sigma)}\right)=-s(\sigma) \mathcal{T}_{-} f,
$$

as desired.

In the case of the modular group, the scattering operator $\mathcal{S}^{\prime}$ can be expressed in terms of the Riemann $\zeta$-function and the gamma function as in (4.7)'. The poles of $S^{\prime}$ are the nontrivial zeroes of $\zeta(1+2 i z)$. Thus if the Riemann hypothesis is true these poles will lie on the $\operatorname{line} \operatorname{Im} z=1 / 4$. A further connection between our theory and the $\zeta$-function can be expressed in terms of the semigroup of operators $Z$ " defined in analogy with $Z$ of $\$ 3$ using $\mathscr{D}_{ \pm}^{\prime \prime}=\mathscr{D}_{ \pm} \cap \mathcal{H}_{E}^{\prime}$ instead of $\mathscr{D}_{ \pm}$. Pavlov and Faddeev [2] have formulated the Riemann hypothesis in terms of properties of $Z^{\prime \prime}$ : The Riemann hypothesis is true if and only if $Z^{\prime \prime}(t)$ decays exponentially. Specifically, in [6] we have shown: The Riemann hypothesis is true if

$$
\lim \sup t^{-1} \log \left\|Z^{\prime \prime}(t) f\right\|_{E}<-1 / 4
$$

for a set of $f$ dense in $\mathcal{H}_{c}^{\prime}$. The relation (6.19) is an assertion about the rate at which energy is propagated to infinity for those solutions of the automorphic 
wave equation which contain no standing waves, i.e. are orthogonal to point eigenvectors. This latter condition prevents the application of hyperbolic techniques for proving local energy decay via nonstandard energy identities.

Another possible approach is to use in (6.19) vectors $f$ obtained by application of $Z^{\prime \prime}(t)$ to $\mathscr{Q}_{-}^{\prime \prime}$; this is discussed in an appendix to $\$ 7$ in [6] entitled "How not to prove the Riemann hypothesis".

Hilbert is reputed to have envisaged a proof of the Riemann hypothesis by constructing a skew-hermitian operator on a Hilbert space whose eigenvalues are of the form $\kappa-1 / 2, \kappa$ being the nontrivial zeroes of $\zeta$. By an analogue of Corollary 4.3 the infinitesimal generator $B^{\prime \prime}$ of $Z^{\prime \prime}$ has as its spectrum the values $\{(\kappa-1) / 2\}$. It follows that

$$
2 B^{\prime \prime}+1 / 2
$$

is the kind of operator on $\mathcal{H}_{c}^{\prime}$ that was envisaged by Hilbert; all that is missing is an appropriate inner product on $\mathcal{H}_{c}^{\prime}$ which makes (6.20) skewhermitian.

7. The point spectrum. In addition to the continuous spectrum analysed in $\S \S 4,5$ and 6, the operator $A$ has a substantial point spectrum. As noted in Remark 6.3, the point spectrum is discrete. In the case of the modular group, analysis of the point spectrum is made particularly easy by exploiting the bilateral symmetry of the fundamental domain $F$ described in (1.10), that is the mapping $x \rightarrow-x$ which takes $F$ into itself. Since the operator $L$ is invariant under the reflection: $x \rightarrow-x$, the domain of $L$ can be reduced to the direct sum of even and odd functions. The boundary conditions induced by a function being automorphic become Dirichlet boundary conditions on half of $F$ for odd functions and Neumann boundary conditions on half of $F$ for even functions. It is not hard to show that under Dirichlet boundary conditions on half of $F$, the proper eigenfunctions form a complete orthonormal system. Thus the point eigenvalues corresponding to odd eigenfunctions of $L$ over $F$ accumulate at infinity. It can also be shown that the number of proper eigenvalues corresponding to even eigenfunctions over $F$ is also infinite. The asymptotic distribution of the proper eigenvalues of $L$ can be related to the asymptotic behavior of the scattering operator, see Theorem 8.6, p. 205 of [6].

Finally we note that if $-\nu^{2}$ is an eigenvalue of $L$ with eigenfunction $p$ :

$$
L p=-\nu^{2} p,
$$

then

$$
g_{ \pm}=\{p, \pm i v p\}
$$

are eigenvectors of $A$ with eigenvalues $\pm i v$ :

$$
A g_{ \pm}= \pm i v g_{ \pm} \cdot
$$

8. The Selberg trace formula. The hyperbolic theory developed here lends itself to two distinct versions of the formula which Selberg derived using the concept of trace for operators of trace class. On the one hand the trace of such an operator is equal to the sum of its eigenvalues; on the other hand when the operator is an integral operator on $L_{2}$ with a smooth kernel, it is 
equal to the integral of the kernel along the diagonal. For Hermitian trace class operators this is standard; the general theorem is due to Lidskii (see [3]). In our first version of the trace formula we need only the Hermitian case, but in our second version we require the general theorem.

We now give a simple illustration of how the trace formula is used. Let $\varphi(t)$ be a real valued smooth even function which decays reasonably fast as $|t| \rightarrow \infty$. Given a positive real number $p$, let $H$ be the space of $p$-periodic functions endowed with the $L_{2}(0, p)$ topology. Finally define $K=K_{\varphi}$ as the convolution operator:

$$
\left(K_{\varphi} f\right)(x)=\int_{-\infty}^{\infty} \varphi(t) f(x-t) d t=\varphi * f .
$$

Clearly $K_{\varphi}$ maps $L_{2}(0, p)$ into itself and is Hermitian. When written as

$$
K_{\varphi} f(x)=\sum_{n} \int_{n p}^{(n+1) p} \varphi(x-t) f(t) d t=\int_{0}^{p} \sum_{n} \varphi(x-t+n p) f(t) d t,
$$

we see that its kernel:

$$
\sum \varphi(x-t+n p)
$$

is smooth; therefore $K_{\varphi}$ is of trace class and its trace is equal to

$$
p \sum \varphi(n p) \text {. }
$$

The eigenfunctions of $K_{\varphi}$ are $\exp (2 \pi i m t / p), m \in \mathbf{Z}$; the eigenvalues are

$$
\lambda_{m}=\int_{-\infty}^{\infty} \varphi(t) \exp \left(\frac{2 \pi i}{p} m t\right) d t=\hat{\varphi}\left(\frac{2 \pi m}{p}\right),
$$

where $\hat{\varphi}$ denotes the Fourier transform of $\varphi$. The trace formula asserts that

$$
p \sum \varphi(n p)=\sum \hat{\varphi}\left(\frac{2 \pi m}{p}\right)
$$

this is the Poisson summation formula.

Returning to the problem at hand, let $U(t)$ be the solution operator for the automorphic wave equation, defined as in $\$ 2$, and let $A$ denote the infinitesimal generator of the group $U$. As we saw earlier, the domain of $A$ can be reduced to two $E$-orthogonal subspaces, one spanned by the proper eigenvectors of $A$ and the other spanned in some sense by the generalized eigenvectors corresponding to the continuous spectrum of $A$. We denote the restrictions of $U$ to these subspaces by $U_{p}$ and $U_{c}$, respectively; clearly

$$
U(t)=U_{p}(t)+U_{c}(t) .
$$

Next we denote by $\mathcal{F}_{1}$ the space of initial data of the form $\{0, f\}$. Note that $E$ is positive definite on $\mathcal{H}_{1}$, in fact $\mathcal{H}_{1}=L_{2}(F)$. Suppose that $u(t)$ is a solution of the automorphic wave equation with initial data in $\mathcal{H}_{1}$, that is $u(0)=0$. Then $-u(-t)$ will have the same initial data as $u(t)$, which shows that $u(t)$ is an odd function of $t$. It follows that $U(t)+U(-t)$ maps $\mathcal{H}_{1}$ into $\mathcal{H}_{1}$.

Let $\varphi$ be a real valued function which is even and decreases exponentially as $|t| \rightarrow \infty$. We define $K=K_{\varphi}$ by

$$
K_{\varphi}=\int_{-\infty}^{\infty} U_{p}(t) \varphi(t) d t
$$


restricted to $\mathcal{H}_{1}$. Clearly $K_{\varphi}$ maps $\mathcal{H}_{1}$ into $\mathcal{H}_{1}$ and is Hermitian.

If $-v_{j}^{2}$ is a negative eigenvalue of $L$ with eigenfunction $p_{j}$, then $\left\{0, p_{j}\right\}$ is an eigenvector of $K_{\varphi}$ with eigenvalue

$$
\kappa_{j}=\int_{-\infty}^{\infty} e^{i \nu_{j} t} \varphi(t) d t=\hat{\varphi}\left(\nu_{j}\right)
$$

as before $\hat{\varphi}$ denotes the Fourier transform of $\varphi$. As we noted earlier, $L$ also has a finite number of positive eigenvalues $\lambda_{j}^{2}, j=1, \ldots, m$ (see (5.1) and (5.2)); these give rise to eigenvalues $\hat{\varphi}\left(i \lambda_{j}\right)$ of $K_{\varphi}$. Note that since $\varphi(t)$ decreases exponentially, $\hat{\varphi}$ is defined for complex arguments.

The estimates on the point eigenvalues of $A$ referred to in $\$ 7$ show that the number of $\nu_{j}$ that do not exceed $\sigma$ is $O\left(\sigma^{2}\right)$. It follows from this that the sum $\Sigma\left|\kappa_{j}\right|$, where $\kappa_{j}$ is given by (8.5), converges provided that $\hat{\varphi}(\tau)$ tends to zero sufficiently fast as $|\tau| \rightarrow \infty$. To this end we further impose some mild assumptions on $\varphi$ and a few of its derivatives. Then $K_{\varphi}$ is of trace class and

$$
\operatorname{tr} K_{\varphi}=\sum \hat{\varphi}\left(\nu_{j}\right)+\sum \hat{\varphi}\left(i \lambda_{j}\right)+\eta \hat{\varphi}(0)
$$

here $\eta$ is the multiplicity of the zero eigenvalue of $L$.

Next in order to evaluate the trace of $K_{\varphi}$ in another way, we express $K_{\varphi}$ as an integral operator. Using (8.3) and (8.4) we write

$$
K_{\varphi}=\int\left[U(t)-U_{c}(t)\right] \varphi(t) d t .
$$

We can express $U_{c}$ in terms of the operational calculus as

$$
U_{c}(t)=\exp A_{c} t
$$

where $A_{c}$ is the absolutely continuous part of $A$. Now in $\S 6$ we have explicity determined the spectral representation of $A_{c}$. The generalized eigenfunctions of $A_{c}$ are given by the extended Eisenstein series $e(w, \sigma)$. Since $A_{c}$ acts as multiplication by $i \sigma$ in the spectral representation, any function $\Phi\left(-i A_{c}\right)$ of $-i A_{c}$ is an integral operator with kernel

$$
\int \Phi(\sigma) e(w, \sigma) e\left(w^{\prime}, \sigma\right) d \sigma .
$$

In particular

$$
\int U_{c}(t) \varphi(t) d t=\int \varphi(t) \exp \left(t, A_{c}\right) d t=\hat{\varphi}\left(-i A_{c}\right)
$$

is an integral operator with kernel

$$
\int \hat{\varphi}(\sigma) e(w, \sigma) e\left(w^{\prime}, \sigma\right) d \sigma .
$$

Next we show how to write $U(t)$ as an integral operator. Denote by $U_{0}(t)$ the solution operator for the non-Euclidean wave operator over all of $\Pi$. Since $L$ is invariant under the non-Euclidean motions, so is $U_{0}(t)$; in particular if $f_{0}$ is automorphic in $\Pi$, so is $U_{0}(t) f_{0}$. Thus if we denote the restriction of $f_{0}$ to $F$ by

$$
f=\chi_{F} f_{0}
$$


then

$$
U(t) f=\chi_{F} U_{0}(t) f_{0}
$$

Since

$$
f_{0}=\sum_{\Gamma} T_{\gamma} f
$$

where

$$
\left(T_{\gamma} f\right)(w)=f(\gamma w)
$$

we conclude that

$$
U(t) f=\chi_{F} \sum_{\Gamma} U_{0} T_{\gamma} f
$$

The non-Euclidean wave equation in $\Pi$ can be solved explicitly: For initial data

$$
u(w, 0)=0, \quad u_{t}(w, 0)=f(w)
$$

we have

$$
u_{t}(w, t)=\frac{\operatorname{sgn} t}{\sqrt{8} \pi} \partial_{t} \int_{r<|t|} \frac{f\left(w^{\prime}\right)}{\sqrt{\cosh t-\cosh r}} d w^{\prime}
$$

where $r$ is the non-Euclidean distance from $w$ to $w^{\prime}$ and $d w^{\prime}$ is the nonEuclidean area element. Using this formula we can represent the restriction of $U_{0}$ to $\mathcal{K}_{1}$ as an integral operator with a distribution kernel $R$, called the Riemann function:

$$
R\left(w, w^{\prime}, t\right)=\frac{\operatorname{sgn} t}{\sqrt{8} \pi} \partial_{t} \frac{1}{\sqrt{\cosh t-\cosh r}} .
$$

Substituting this into (8.9) we see that the kernel of $\int U(t) \varphi(t) d t$ is

$$
\frac{1}{\sqrt{8} \pi} \int \varphi(t) \operatorname{sgn} t \partial_{t} \sum_{\Gamma} \frac{1}{\sqrt{\cosh t-\cosh r_{\gamma}}} d t \text {. }
$$

Following Selberg we separate the sum in this integral into four parts corresponding to the identity, the hyperbolic, the elliptic and the parabolic elements of $\Gamma$. Within each part we group the terms into conjugacy classes of $\Gamma$. Accordingly, (8.11) is broken into the sum of four kernels, three of which are of trace class. The trace of the part coming from the identity is

$$
-\frac{\operatorname{area}(F)}{4 \pi} \int \frac{\varphi^{\prime}(t)}{\sinh t / 2} d t \text {. }
$$

For the modular group area $(F)=\pi / 3$. The trace of the part coming from the hyperbolic elements is the weighted sum

$$
\sum_{\tau} \sum_{k \neq 0} \frac{l}{2|\sinh (k l / 2)|} \varphi(k l)
$$

where the first sum is over all inequivalent primitive hyperbolic elements $\tau$ of $\Gamma$, and $l=l(\tau)$ is defined by

$$
2 \cosh l=\operatorname{tr} \tau .
$$


The trace of the part coming from the elliptic elements is a sum of weighted integrals:

$$
\sum_{\tau} \sum_{1}^{m-1} \int \varphi(t) \frac{\cosh t / 2}{\sinh ^{2} t / 2+\sin ^{2} k \pi / m} d t
$$

where the first sum is over all inequivalent primitive elliptic elements $\tau$ and $m=m(\tau)$ is the order of $\tau$, i.e. the smallest $m$ such that $\tau^{m}=I$. Alternatively

$$
2 \cos \pi / m=\operatorname{tr} \tau \text {. }
$$

The part corresponding to the parabolic elements is not of trace class, but its difference from $\hat{\varphi}\left(-i A_{c}\right)$ is. Using (8.8) the trace of the difference can be expressed as a weighted integral of $\hat{\varphi}$; for the modular group this difference is the sum of two terms:

$$
\frac{1}{4} \hat{\varphi}(0)-\log 2 \varphi(0)-\frac{1}{2 \pi} \int \hat{\varphi}(\sigma) \frac{d}{d \sigma} \log \Gamma(1+i \sigma) d \sigma \quad(8.12)_{\mathrm{par}}
$$

and

$$
\frac{1}{4} \hat{\varphi}(0)+\frac{1}{4 \pi} \int \hat{\varphi}(\sigma) \frac{1}{i} \frac{d}{d \sigma} \log s(\sigma) d \sigma ;
$$

here $s(\sigma)$ is the scattering function appearing in formulas (4.7) and (6.18). For arbitrary discrete subgroups (with noncompact fundamental domain of finite area) $s(\sigma)$ has to be replaced by the determinant of the so-called scattering matrix, and the three terms in (8.12) par $_{\text {ave }}$ ho be multiplied by the number of cusps, i.e. points at infinity, of the fundamental domain (which is the same as the number of inequivalent primitive parabolic elements in $\Gamma$ ). The trace formula says that

$$
(8.6)=(8.12)_{\mathrm{id}}+(8.12)_{\mathrm{hyp}}+(8.12)_{\mathrm{ell}}+(8.12)_{\mathrm{par}}+(8.12)_{\mathrm{cont}} \text {. }
$$

For further details we refer to $\$ 9$ of [6] and to Kubota [4].

It should be remarked that a similar trace formula has been derived by Selberg for discrete subgroups whose fundamental domain $F$ is compact. The formula and its derivation are simpler in this case since there is no continuous spectrum and since $\Gamma$ contains no parabolic elements; see [9] for an illuminating exposition and a geometrical interpretation of the trace formula.

We remark finally that when the procedure described above is applied to the trivial case of the classical wave equation for functions of $x$ and $t$ which are periodic in $x$, the operator (8.4) reduces to the operator $(8.1)$ and, as remarked earlier, the trace formula becomes the Poisson summation formula.

We turn now to a description, even sketchier than the foregoing, of our other approach to the Selberg trace formula; this is described more fully in an appendix to $\$ 9$ of [6]. It is based on the notion of trace for non-Hermitian operators.

Let $Z(t)$ denote the semigroup of operators

$$
Z(t)=P_{+} U(t) P_{-},
$$

where, as in $\S 3, P_{ \pm}$are the orthogonal projections onto $\mathscr{D}_{ \pm}^{ \pm}$and $U(t)$ is as before; we take as the domain of $Z(t)$ all of the original Hilbert space $\mathcal{H}_{G}$. As so defined $Z(t)$ does not converge strongly to the identity as $t \rightarrow 0$; however 
the restriction to $\mathscr{K}=\mathscr{H}_{G} \ominus\left(\mathscr{D}_{+} \oplus \mathscr{D}_{-}\right)$does have this property. We take a function $\varphi(t)$ of compact support in $\mathbf{R}_{+}$and define $C=C_{\varphi}$ by

$$
C_{\varphi}=\int_{0}^{\infty} Z(t) \varphi(t) d t .
$$

We claim that $C_{\varphi}$ is of trace class. For proof we refer to [6], where two demonstrations are given. In the second proof, contained in Appendix 2 to $\$ 9$, it is shown that $C_{\varphi}$ is an integral operator whose kernel is smooth except for a jump dicontinuity along the line $y=a$ (caused by the action of $P_{ \pm}$) and that the kernel decays faster than any power of $y$ as $y \rightarrow \infty$. The argument showing this decay is based, appropriately enough, on the Poisson summation formula.

We now determine the eigenvectors of $C_{\varphi}$. Aside from the null vectors of $C_{\varphi}$, which do not contribute to the trace, all of these are eigenvectors of $Z(t)$ restricted to $\mathscr{K}$. It is shown in the appendix to Chapter 9 of [6] that these eigenvectors are obtained, by $P_{+}$projection, from the eigenvectors of $U(t)$ which are orthogonal to $\mathscr{D}_{\ominus}$.

We treat first the proper eigenvectors of $U(t)$. We saw in $\$ 7$ that $L$ has infinitely many eigenfunctions $p$ corresponding to negative eigenvalues $-\nu^{2}$ :

$$
L p=-\nu^{2} p \text {. }
$$

Integrating this relation with respect to $x$, we get for the zero Fourier coefficient $p^{(0)}$ the ordinary differential equation:

$$
y^{2} \frac{d^{2}}{d y^{2}} p^{(0)}+\frac{1}{4} p^{(0)}=-\nu^{2} p(0),
$$

all of whose solutions are of the form

$$
p^{(0)}=a y^{1 / 2-i \nu}+b y^{1 / 2+i \nu} .
$$

Since $-\nu^{2}$ is in the point spectrum, $p$ belongs to $L_{2}(F)$ and this requires both $a$ and $b$ to be zero, i.e. $p^{(0)}=0$.

We saw in $\$ 7$ that if $p$ is an eigenfunction of $L$, then

$$
g_{ \pm}=\{p, \pm i v p\}
$$

are eigenvectors of $U(t)$, with eigenvalues $\exp ( \pm i \nu t)$. The condition $p^{(0)}=0$ implies that $g_{ \pm}$are orthogonal to both $\mathscr{D}_{-}$and $\mathscr{D}_{+}$, i.e. that

$$
P_{-} g_{ \pm}=P_{+} g_{ \pm}=g_{ \pm} \text {. }
$$

It follows that $g_{ \pm}$are eigenvectors of $Z(t)$ as well, with eigenvalues $\exp ( \pm i v t)$; hence $g_{ \pm}$are also eigenvectors of $C_{\varphi}$, with eigenvalues

$$
\hat{\varphi}\left( \pm \nu_{j}\right) \text {. }
$$

We have noted in $\S 5$ that $L$ has a finite number of positive eigenvales $\lambda^{2}$; denote the corresponding eigenfunction by $q$. Then $\{q, \lambda q\}$ is an eigenvector of $U(t)$, with eigenvalue $\exp (\lambda t)$. As remarked in (5.6), for $\lambda>0,\{q, \lambda q\}$ is orthogonal to $\mathscr{D}_{-}$; from this one can deduce, using the relation (3.7), that $P_{+}\{q, \lambda q\}$ is an eigenvector of $Z(t)$ with eigenvalue $\exp (\lambda t)$, and therefore of $C_{\varphi}$ with eigenvalue

$$
\hat{\varphi}\left(-i \lambda_{j}\right)
$$


The vector $\{q,-\lambda q\}$ is also an eigenvector of $U(t)$, in this case orthogonal to $\mathscr{D}_{+}$, but not in general orthogonal to $\mathscr{D}_{-}$. However if it happens also to be orthogonal to $\mathscr{Q}_{-}$, then as above $\{q,-\lambda q\}$ is an eigenvector of $Z(t)$ with eigenvalue $\exp (-\lambda t)$, and of $C_{\varphi}$ with eigenvalue

$$
\hat{\varphi}\left(i \lambda_{j}\right) \text {. }
$$

We turn now to the generalized eigenvectors of $U(t)$ which are orthogonal to $\mathscr{D}_{-}$. The corresponding eigenvalues appeared in the proof of Theorem 4.2 as poles of the meromorphic continuation of the scattering function $s(z)$ into the upper half plane. According to this theorem, these poles can occur only at points of $-i \sigma(B)$; here $B$ denotes the infinitesimal generator of $Z(t)$ restricted to $\mathcal{H}$. Taking the residue of the Eisenstein series about such a point, it is clear from (4.7) that the resulting generalized eigenvector of $A$ will be orthogonal to $\mathscr{D}_{-}$and hence, after projection by $P_{+}$, will be a proper eigenvector of $B$. The corresponding eigenvalue of $B$ is a complex number $\mu_{j}, \operatorname{Re} \mu_{j}<0$, that of $Z(t)$ is $\exp \left(\mu_{j} t\right)$ and that of $C_{\varphi}$ is

$$
\hat{\varphi}\left(-i \mu_{j}\right) \text {. }
$$

Finally we denote by $\eta_{0}$ the multiplicity of 0 as an eigenvalue of $B$; then $\hat{\varphi}(0)$ is the corresponding eigenvalue of $C_{\varphi}$ and it has multiplicity $\eta_{0}$. Thus the sum of the eigenvalues of $C_{\varphi}$ is obtained by adding (8.17) to (8.18), (8.18)', (8.19) and to the above. Altogether we get

$$
\sum \hat{\varphi}\left( \pm \nu_{j}\right)+\sum \hat{\varphi}\left(-i \lambda_{j}\right)+\Sigma^{\prime} \hat{\varphi}\left(i \lambda_{j}\right)+\sum \hat{\varphi}\left(-i \mu_{j}\right)+\eta_{0} \hat{\varphi}(0)
$$

Next we construct the kernel of $C_{\varphi}$ as integral operator. This task is roughly similar to constructing the kernel of $K_{\varphi}$, mildly complicated by the fact that (i) $C_{\varphi}$ acts on vectors with two components, and (ii) the underlying Hilbert space is the direct sum of $L_{2}(F)$ and a Dirichlet space over $F$. When the dust settles, we end up with the following formula:

$$
(8.20)=\hat{\varphi}(0) / 2+(8.12)_{\mathrm{id}}+(8.12)_{\mathrm{hyp}}+(8.12)_{\mathrm{ell}}+(8.12)_{\mathrm{par}} \text {. }
$$

It is instructive to compare this with our previous trace formula. We extend $\varphi$ used in the definition of $C_{\varphi}$ in (8.16) as an even function of $t$ and use this extended $\varphi$ in the definition of $K_{\varphi}$ in (8.7). Subtracting the trace formula for $K_{\varphi}$ from that for $C_{\varphi}$ we obtain an identity of the following form

$$
\begin{aligned}
\sum \hat{\varphi}\left(-i \mu_{j}\right) & -\sum^{\prime \prime} \hat{\varphi}\left(i \lambda_{j}\right)+\left(\eta_{0}-2 \eta\right) \hat{\varphi}(0) \\
& =\frac{i}{4 \pi} \int[\hat{\varphi}(\sigma)+\hat{\varphi}(-\sigma)] \frac{d}{d \sigma} \log s(\sigma) d \sigma .
\end{aligned}
$$

Since the poles of $s(z)$ in the upper half plane coincide with the set $-i \mu_{j}$ and the so-called relevant $i \lambda_{j}$ 's, this formula can be verified by deforming the integral on the right from the real axis off to infinity. This can be justified on the basis of appropriate estimates for $s(z)$ away from the poles; for the modular group such estimates are available. In order to verify (8.21) in general we use a "mini-trace formula" for the operator

$$
C_{\varphi}^{\prime \prime}=\int Z^{\prime \prime}(t) \varphi(t) d t
$$


on $\mathcal{K}^{\prime \prime}$, where

$$
\mathscr{K}^{\prime \prime}=\mathcal{H}_{c}^{\prime} \ominus\left(\mathscr{Q}_{-}^{\prime \prime} \oplus \mathscr{W}_{+}^{\prime \prime}\right)
$$

and

$$
Z^{\prime \prime}(t)=P_{+}^{\prime \prime} U(t) P_{-}^{\prime \prime} ;
$$

here $\mathscr{Q}_{ \pm}^{\prime \prime}$ denote that subspace of $\mathscr{Q}_{ \pm}$which is orthogonal to the subspace $\mathscr{P}$ defined in (5.4) and $P_{ \pm}^{\prime \prime}$ are orthogonal projections onto $\mathcal{H}_{c}^{\prime} \ominus \mathscr{Q}_{ \pm}^{\prime \prime}$. As a by-product of this analysis we find that $\eta_{0}=2 \eta$. For details see the appendix to $\$ 9$ of [6].

Appendix: A new proof of Theorem 2.1. Theorem 2.1 is a restatement of Theorem 6.6 in [6]. Since the initial steps of the two proofs are the same we shall refer the reader to [6, pp. 126-130] for the proofs of some of the initial lemmas.

THEOREM 2.1. For every $\lambda$ in the resolvent set of $A,(\lambda I-A)^{-1}$ maps the unit ball in $\mathcal{K}$ into a compact subset of $\mathcal{H}_{G}$.

REMARK. Since it suffices to prove the theorem for a single value of $\lambda$, we shall for convenience take $\lambda$ to be real $>4$.

Theorem 2.1 is a consequence of a simpler result:

TheOREM A.1. Let $\varphi$ be any $C_{0}^{\infty}$ function on $\mathbf{R}_{+}$. Define the operator $M=M_{\varphi} b y$

$$
M_{\varphi}=\int_{0}^{\infty} \varphi(t) U(t) d t .
$$

Then $M_{\varphi}$ maps the unit ball in $\mathscr{K}$ into a compact subset of $\mathcal{H}_{G}$.

The proof of this theorem is based on the following two propositions:

LEMMA A.2. (a) The set

$$
u=M f, \quad G(f) \leqslant 1,
$$

is precompact with respect to the norm

$$
G_{Y}(u)=\int_{F(Y)}\left\{\left|u_{1, x}\right|^{2}+\left|u_{1, y}\right|^{2}+\left|u_{1}\right|^{2}+\left|u_{2}\right|^{2}\right\} d x d y
$$

for any $Y$; here $F(Y)$ denotes the domain

$$
F(Y)=F \cap\{y<Y\} \text {. }
$$

(b) Given any $\varepsilon>0$, there is a $Y>$ a such that

$$
G^{Y}(u)=\int_{y>Y}\left\{\left|u_{1, x}\right|^{2}+y\left|\partial_{y} \frac{u_{1}}{\sqrt{y}}\right|^{2}+\frac{\left|u_{2}\right|^{2}}{y^{2}}\right\} d x d y \leqslant \varepsilon G(f)
$$

for all $u$ of the form (A.2) with $f$ in $\mathscr{K}$.

Part (a) is a simple consequence of Rellich's compactness criterion.

ProOF OF PART (b). We note that the imposition of a finite number of linear continuous constraints on the domain of $M$ does not affect the precompactness argument. Now $f_{1}^{(0)}(a)=0$ is just such a constraint since by Lemma 4.2 
in [6]

$$
\left|f_{1}^{(0)}(a)\right|^{2} \leqslant \int_{-1 / 2}^{1 / 2}\left|f_{1}(x, a)\right|^{2} d x \leqslant \text { const } G(f) .
$$

Since for $f$ in $\mathscr{K}, f_{2}^{(0)}(y)=0$ for all $y>a$, it follows by (2.17) that we can assume without loss of generality that $f^{(0)}(y)=0$ for all $y>a$.

Fixing $\varphi$, we choose $T$ so that supp $\varphi \subset(0, T)$. Since the speed of propagation for the solution to the non-Euclidean wave equation is $\leqslant 1$, a signal which originates at a point below $y=2 b e^{T}$ (or above $y=b e^{T}$ ) will not get above $y=2 b e^{2 T}$ (or below $y=b$ ) during time $t \in(0, T)$. According to (A.1) and (A.2), $u$ is a superposition of values of $U(t) f, 0<t<T$. It follows that values of $u$ at points where $y>2 b e^{2 T}$ (or $y<b$ ) do not depend on values of $f$ below $y=2 b e^{T}$ (or above $y=b e^{T}$ ). This suggests that we split $f$ as

$$
f=g+h
$$

so that

$$
\begin{array}{ll}
g=0 & \text { for } y<b e^{T} \\
h=0 & \text { for } y>2 b e^{T}
\end{array}
$$

and so that

$$
G(g) \leqslant \text { const } G(f) \text {. }
$$

We can do this in such a way that the constant is independent of our choice of $b>a$, which will be fixed later on.

Since part (b) of the proof is concerned only with values of $u$ for large $y, h$ plays no role in what follows. We therefore need only consider $g$ and to keep our notation simple we shall hereafter denote $g$ by $f$. We may therefore assume that $f$ satisfies

$$
f=0 \text { for all } y<b e^{T} .
$$

In this case $u=0$ for all $y<b$. Moreover the various Fourier coefficients of $u$, that is the

$$
u^{(k)}(y)=\int_{-1 / 2}^{1 / 2} e^{-i k x} u(x, y) d x
$$

are obtained directly from $M$ acting only on the corresponding Fourier coefficients of $f$; that is, the Fourier coefficients of $U(t) f$ remain uncoupled as long as the signal stays above $y=a$ as it does for $0<t<T$. In particular $u^{(0)}=0$.

We note that $G$ and $E$ are identical for data vanishing for $y<a$. Since $f^{(0)}=0$ for all $y$, we have

$$
\begin{aligned}
G(f) & =\int_{a}^{\infty} \int_{-1 / 2}^{1 / 2}\left\{\left|\partial_{x} f_{1}\right|^{2}+y\left|\partial_{y} \frac{f_{1}}{\sqrt{y}}\right|^{2}+\frac{\left|f_{2}\right|^{2}}{y^{2}}\right\} d x d y \\
& =\sum_{k \neq 0} \int_{a}^{\infty}\left\{(2 \pi k)^{2}\left|f_{1}^{(k)}\right|^{2}+y\left|\partial_{y} \frac{f_{1}^{(k)}}{\sqrt{y}}\right|^{2}+\frac{\left|f_{2}^{(k)}\right|^{2}}{y^{2}}\right\} d y .
\end{aligned}
$$


We now set

$$
p=(\lambda I-A) u=\int\left(\lambda \varphi+\partial_{t} \varphi\right) U(t) f d t
$$

Clearly

$$
G(p) \leqslant \text { const } G(f)
$$

Further writing (A.8) in component form, we get

$$
\lambda u_{1}-u_{2}=p_{1}, \quad \lambda u_{2}-L u_{1}=p_{2}
$$

so that

$$
\lambda^{2} u_{1}-y^{2} \Delta u_{1}-u_{1} / 4=\lambda p_{1}+p_{2} \equiv q .
$$

Applying (A.7) to $p_{1}$ we get

$$
\sum_{k \neq 0} \int_{b}^{\infty}\left|p_{1}^{(k)}\right|^{2} d y \leqslant G(p)
$$

and combining this with (A.9) we see that

$$
\sum_{k \neq 0} \int_{b}^{\infty} \frac{\left|q^{(k)}\right|^{2}}{y^{2}} d y \leqslant \text { const } G(p) \leqslant \text { const } G(f) .
$$

Finally it is clear from (A.8)" that the $k$ th Fourier coefficient of $u_{1}$ satisfies the equation

$$
-\frac{d^{2}}{d y^{2}} u_{1}^{(k)}+\left(4 \pi^{2} k^{2}+\frac{\kappa}{y^{2}}\right) u_{1}^{(k)}=\frac{q^{(k)}}{y^{2}},
$$

where $\kappa=\lambda^{2}-1 / 4$.

Lemma A.3. Let $v \in C^{1}(\mathbf{R})$ and $\int_{a}^{\infty}|v|^{2} d y<\infty$, then there exists a sequence $y_{j} \rightarrow \infty$ for which $\left.\operatorname{Re} v^{\prime} \bar{v}\right|_{y_{j}} \rightarrow 0$.

Proof. Obviously

$$
\lim _{y \rightarrow \infty} \int_{j}^{j+1}|v|^{2} d y=0 .
$$

By the law of the mean there is $y_{j}^{\prime}$ in $(j, j+1)$ for which $v\left(y_{j}^{\prime}\right) \rightarrow 0$. Moreover

$$
\left|v\left(y_{j+2}^{\prime}\right)\right|^{2}-\left|v\left(y_{j}^{\prime}\right)\right|^{2}=\int_{y_{j}^{\prime}}^{y_{j+2}^{\prime}} \frac{d}{d y}|v|^{2} d y=2 \int_{y_{j}^{\prime}}^{y_{j+2}^{\prime}} \operatorname{Re}\left(v^{\prime} \bar{v}\right) d y .
$$

Since this expression converges to zero and since $y_{j+2}^{\prime}-y_{j}^{\prime} \geqslant 1$, the law of the mean again furnishes us with a $y_{j}$ in $\left(y_{j}^{\prime}, \mathrm{y}_{j+2}^{\prime}\right)$ satisfying the statement of the lemma.

We return now to the proof of part (b) of Lemma A.2. Multiply the relation (A.12) by $\overline{u_{1}^{(k)}}$ and integrate by parts. Take the real part of the resulting relation and use Lemma A.3 at the upper limit of integration and the fact $u_{1}^{(k)}(b)=0$ at the lower limit. This gives us

$$
\int_{b}^{\infty}\left\{\left|u_{1}^{(k)^{\prime}}\right|^{2}+\left(4 \pi^{2} k^{2}+\frac{\kappa}{y^{2}}\right)\left|u_{1}^{(k)}\right|^{2}\right\} d y=2 \operatorname{Re} \int_{b}^{\infty} \frac{q^{(k)}}{y^{2}} \overline{u_{1}^{(k)}} d y .
$$


Applying the Schwarz inequality to the right member gives us

$$
\int_{b}^{\infty}\left\{\left|u_{1}^{(k)^{\prime}}\right|^{2}+4 \pi^{2} k^{2}\left|u_{1}^{(k)}\right|^{2}\right\} d y \leqslant \frac{1}{b^{2}} \int_{b}^{\infty} \frac{\left|q^{(k)}\right|^{2}}{y^{2}} d y+\int_{b}^{\infty}\left|u_{1}^{(k)}\right|^{2} d y
$$

and transposing the last term on the right we get

$$
\int_{b}^{\infty}\left\{\left|u_{1}^{(k)^{\prime}}\right|^{2}+2 \pi^{2} k^{2}\left|u_{1}^{(k)}\right|^{2}\right\} d y \leqslant \frac{1}{b^{2}} \int_{b}^{\infty} \frac{\left|q^{(k)}\right|^{2}}{y^{2}} d y .
$$

From the first relation in (A.8) we get

$$
\begin{aligned}
\int_{b}^{\infty} \frac{\left|u_{2}^{(k)}\right|^{2}}{y^{2}} d y & \leqslant 2 \lambda^{2} \int_{b}^{\infty} \frac{\left|u_{1}^{(k)}\right|^{2}}{y^{2}} d y+2 \int_{b}^{\infty} \frac{\left|p_{1}^{(k)}\right|^{2}}{y^{2}} d y \\
& \leqslant \frac{2}{b^{2}}\left[\lambda^{2} \int_{b}^{\infty}\left|u_{1}^{(k)}\right|^{2} d y+\int_{b}^{\infty}\left|p_{1}^{(k)}\right|^{2} d y\right] .
\end{aligned}
$$

It now follows from

$$
\begin{aligned}
\int_{b}^{\infty} y\left|\partial_{y} \frac{u_{1}^{(k)}}{\sqrt{y}}\right|^{2} d y & \leqslant 2 \int_{b}^{\infty} \frac{\left|u_{1}^{(k)}\right|^{2}}{y^{2}} d y+2 \int_{b}^{\infty}\left|u_{1}^{(k)^{\prime}}\right|^{2} d y \\
& \leqslant 2 \int_{b}^{\infty}\left(\left|u_{1}^{(k)}\right|^{2}+\left|u_{1}^{(k)^{\prime}}\right|^{2}\right) d y
\end{aligned}
$$

together with (A.7) as applied to $u_{1}$, (A.10), (A.11), (A.13) and (A.14) that

$$
G(u) \leqslant \frac{\text { const }}{b^{2}} G(f),
$$

where the constant is independent of our choice of $b$. Hence if we choose $b$ so that const $/ b^{2}<\varepsilon$ and $Y>2 b e^{2 T}$, then we get the desired inequality for part (b) of Theorem A.1.

\section{REFERENCES}

1. L. D. Faddeev, Expansion in eigenfunctions of the Laplace operator in the fundamental domain of a discrete group on the Lobacevskii plane, Trudy Moscov. Mat. Obšč. 17 (1967), 323-350; see also English transl., Trans. Moscow Math. Soc. 17 (1967), 357-386.

2. L. D. Faddeev and B.S. Pavlov, Scattering theory and automorphic functions, Proc. Steklov Inst. Math. 27 (1972), 161-193.

3. I. C. Gohberg and M. G. Krein, Introduction to the theory of linear non-selfadjoint operators, Transl. Math. Monographs, vol. 18, Amer. Math. Soc., Providence, R. I., 1969.

4. T. Kubota, Elementary theory of Eisenstein series, Wiley, New York, 1973.

5. P. D. Lax and R. S. Phillips, Scattering theory, Academic Press, New York, 1967.

6. Scattering theory for automorphic functions, Ann. of Math. Studies, no. 87, Princeton Univ. Press, Princeton, N. J., 1976.

7. __ The scattering of sound waves by an obstacle, Comm. Pure Appl. Math. 30 (1977), 195-233.

8. ,Translation representations for the solution of the non-Euclidean wave equation, Comm. Pure Appl. Math. 32 (1979), 617-667. 
9. H. P. McKean, Selberg's trace formula as applied to a compact Riemann surface, Comm. Pure Appl. Math. 25 (1972), 225-246.

10. A. Selberg, Harmonic analysis and discontinuous groups in weakly symmetric Riemannian spaces with applications to Dirichlet series, J. Indian Math. Soc. 20 (1956), 47-87.

11. M. A. Semenov-Tian-Shansky, Harmonic analysis on Riemannian symmetric spaces of negative curvature and scattering theory, Math. USSR Izvestija, vol. 10 (1976), 535-563.

Courant Institute of Mathematical Sciences, New York University, New York, New YoRK 10012

Department of Mathematics, Stanford University, Stanford, California 94305 
